\title{
Existence of financial equilibria with restricted participation
}

\author{
Zaier Aouani ${ }^{\mathrm{a}}$, Bernard Cornet ${ }^{\mathrm{b}}$ \\ ${ }^{a}$ International School of Economics at TSU, Tbilisi, Georgia. aouani@gmail.com, aouani@iset.ge \\ ${ }^{b}$ University of Kansas, Lawrence, Kansas and Université Paris 1 Panthéon-Sorbonne, Paris, France. cornet@ku.edu, \\ cornet@univ-paris1.fr
}

\begin{abstract}
We consider a two-date model of a financial exchange economy with finitely many agents having nonordered preferences and portfolio constraints. There is a market for physical commodities at any state today or tomorrow and financial transfers across time and across states are allowed by means of finitely many nominal assets or numéraire assets. We prove a general existence result of equilibria for such a financial exchange economy in which portfolios are defined by linear constraints, extending the framework of linear equality constraints by Balasko et al. (1990), and the existence results in the unconstrained case by Cass (1984, 2006), Werner (1985), Duffie (1987), and Geanakoplos and Polemarchakis (1986). Our main result is a consequence of an auxiliary result, also of interest for itself, in which agents' portfolio constraints are defined by general closed convex sets and the financial structure is assumed to satisfy a "nonredundancy-type" assumption, weaker than the ones in Radner (1972) and Siconolfi (1989).
\end{abstract}

Keywords: Restricted participation, portfolio constraints, financial exchange economy, equilibrium, existence, arbitrage-free asset prices

JEL: C62, D52, D53

\section{Introduction}

Since the seminal paper by Radner (1972) proving the existence of equilibria in a financial exchange economy with bounded portfolio sets, and the non-existence issue raised by Hart (1975), Duffie and Shafer $(1985,1986)$ showed a generic existence result with real assets. An extensive body of literature built upon their argument, see e.g. Geanakopolos and Shafer (1990), Hirsch et al. (1990), Husseini et al. (1990) and Bich and Cornet $(2004,2009)$. Subsequently, the literature on the existence problem paid particular attention to incomplete asset markets with only nominal assets or only numéraire assets; this was considered either in the case of unconstrained agents' portfolio holdings, e.g. Cass (1984, 2006), Werner (1985), or Duffie (1987) for nominal assets and Geanakoplos and Polemarchakis (1986) for numéraire assets, as well as when agents' participation to financial markets might be restricted.

With restricted participation, in addition to the budget constraint, each agent $i$ faces exogenous portfolio constraints $z_{i} \in Z_{i} \subset \mathbb{R}^{J}$, where $J$ denotes the (finite) number of assets in the economy. The presence of such portfolio constraints is a natural cause of market incompleteness and allows to capture a wide range

October 18, 2009 
of imperfections in the financial markets, such as short selling constraints, collateral requirements, and more generally institutional constraints. Elsinger and Summer (2001) give an extensive discussion of these institutional constraints and how to model them in a general financial framework. The existence problem had recently a growing interest since the first work by Siconolfi (1989), and Cass (1984, 2006). Linear equality constraints are considered by Balasko et al. (1990) with nominal assets, and by Polemarchakis and Siconolfi (1997) with real assets. More recently, the case of portfolio sets $Z_{i}$ which are closed, convex subsets containing zero as in Siconolfi (1989) is considered by Angeloni and Cornet (2006) for real assets and by Martins-da-Rocha and Triki (2005), Hahn and Won (2007), and Cornet and Gopalan (2010) in the nominal case.

This paper considers a two-date stochastic model $(t=0$ and $t=1)$ of a financial exchange economy with finitely many states of nature, one of which is revealed at $t=1$. There is a market for finitely many physical goods at every state today or tomorrow and financial transfers across time and across states are allowed by means of finitely many assets. There are finitely many agents with non-ordered preferences and portfolio constraints described by closed, convex subsets containing zero. Our contribution is twofold. First, when financial assets are nominal or numéraire, we provide a general existence result of equilibria (Theorem 1). Apart from standard assumptions on the consumption side (preferences and endowments), we assume that portfolio restrictions are defined by linear inequality constraints. This extends the framework of linear equality constraints considered by Balasko et al. (1990), and the standard model of unconstrained portfolios. Our existence result generalizes previous work by Cass (1984), Werner (1985), Duffie (1987), and Geanakoplos and Polemarchakis (1986).

Our second contribution provides an auxiliary result (Theorem 2) which is the key tool in the proof of Theorem 1. In this auxiliary result, we make an additional "nonredundancy-type" (or "reduced form") assumption (F3 in the text) on the financial side. In the case of nominal assets and no restrictions on portfolio trades $\left(Z_{i}=\mathbb{R}^{J}\right.$ for all $\left.i\right)$, Assumption $\mathbf{F 3}$ is equivalent to the fact that the payoff matrix $V$ has no redundant assets, that is, $\operatorname{rank} V=J$ or equivalently $\operatorname{ker} V=\{0\}$. In this case there is a priori no loss of generality in assuming that there are no redundant assets, otherwise, by deleting the redundant columns we obtain a "reduced" financial economy, whose equilibria yield equilibria in the original one. However, as mentioned in Balasko et al. (1990), one significant source of restricted participation is financial intermediation which typically involves redundancy. So there is no a priori grounds for the standard Full Rank Assumption in the presence of restricted participation, which therefore will be superseded by Assumption F3.

In the case of linear equality portfolio constraints (i.e., the $Z_{i}$ 's are vector spaces), Balasko et al. (1990) show how to transform the agents' financial opportunities to obtain a "reduced" financial economy in which each agent's portfolio choice is a subspace having the same dimension as the wealth space it generates, that is, $Z_{i} \cap \operatorname{ker} V=\{0\}$ for all $i$ (Siconolfi (1989)); moreover, every equilibrium in the "reduced" economy leads to an equilibrium in the original economy. In this paper, we extend the analysis to the case of linear inequality constraints with nominal or numéraire assets. We show how to "reduce" the financial structure to obtain a new financial structure satisfying Assumption F3, a weaker condition than Siconolfi (1989)'s, 
keeping the correspondence between the equilibria; moreover, Assumption F3 coincides with Siconolfi's when the $Z_{i}$ 's are linear subspaces. Finally, we mention the companion papers Aouani and Cornet $(2008 \mathrm{a}, \mathrm{b})$ and the paper by Hahn and Won (2007), which study the more general case of closed convex portfolio sets.

The paper is organized as follows. In Section 2, we describe the financial exchange economy, we state our main existence result (Theorem 1) in the case of nominal or numéraire assets, and we state the auxiliary result (Theorem 2) under the additional Assumption F3. This section also provides examples under which Assumption F3 and the Financial Survival Assumption are satisfied. In Section 2.6, the proof of our main result (Theorem 1) is given as a consequence of Theorem 2 by "reducing" the initial economy into a new economy satisfying Assumption F3 whose equilibria yield equilibria in the original one. Finally, we also discuss the relationship with the existence results in the literature by Radner (1972), Siconolfi (1989), Cass (1984, 2006), Werner (1985), Duffie (1987) and Geanakoplos and Polemarchakis (1986). Section 3 is devoted to the proof of the auxiliary result (Theorem 2). The Appendix gathers the proofs of some lemmata used in the proofs of Theorems 1 and 2.

\section{The model and the main result}

\subsection{The stochastic financial exchange economy}

${ }^{1}$ The stochastic model considers two dates: $t=0$ (today) and $t=1$ (tomorrow). At the second date, there is a nonempty finite set $\mathcal{S}:=\{1, \ldots, S\}$ of states of nature, one of which prevails at time $t=1$ and is only known at time $t=1$. For convenience, $s=0$ denotes the state of the world (known with certainty) at period 0 and we let $\overline{\mathcal{S}}=\{0\} \cup \mathcal{S}=\{0,1, \ldots, S\}$. At each state, today and tomorrow, there is a spot market for a positive number $\ell$ of divisible physical goods and we assume that the goods are perishable, i.e., each good does not last more than one period. In this model a commodity is a couple $(h, s)$, specifying the physical $\operatorname{good} h=1, \ldots, \ell$ and the state $0,1, \ldots, S$ at which it is available. Thus the commodity space is $\mathbb{R}^{L}$, where $L=\ell(1+S)$. An element $x$ (resp. $p$ ) in $\mathbb{R}^{L}$ is called a consumption (resp. a price) and we will use the notation $x=(x(s))_{s \in \overline{\mathcal{S}}} \in \mathbb{R}^{L}$, where $x(s)=\left(x_{1}(s), \ldots, x_{\ell}(s)\right) \in \mathbb{R}^{\ell}$, denotes the spot consumption at node $s \in \overline{\mathcal{S}}$.

\footnotetext{
${ }^{1}$ We shall use hereafter the following notations. If $\mathcal{I}$ is a finite set, whose number of elements is $I$, the space $\mathbb{R}^{I}$ (identified to the space $\mathbb{R}^{I}$ of functions $x: I \rightarrow \mathbb{R}$ whenever necessary) is endowed with the scalar product $x \cdot y:=\sum_{i=1}^{I} x_{i} y_{i}$, and we denote by $\|x\|:=\sqrt{x \cdot x}$ the Euclidean norm, $B_{I}(x, r):=\left\{y \in \mathbb{R}^{I}:\|y-x\| \leq r\right\}$, the closed ball centered at $x \in \mathbb{R}^{I}$ of radius $r>0$. For $x=\left(x_{i}\right)$ and $y=\left(y_{i}\right)$ in $\mathbb{R}^{I}$, the notation $x \geq y$ (resp. $x>y, x \gg y$ ) means that, for every $i, x_{i} \geq y_{i}$ (resp. $x \geq y$ and $x \neq y$, resp. $\left.x_{i}>y_{i}\right)$ and we let $\mathbb{R}_{+}^{I}=\left\{x \in \mathbb{R}^{I}: x \geq 0\right\}, \mathbb{R}_{++}^{I}=\left\{x \in \mathbb{R}^{I}: x \gg 0\right\}$. Let $X \subset \mathbb{R}^{I}$, the span of $X$ is the linear subspace of $\mathbb{R}^{I}$, denoted $\langle X\rangle$, which is the set of all the $K$-linear combinations $\sum_{k=1}^{K} \alpha_{k} x_{k}$ of vectors $x_{k} \in X$ for every integer $K$, and we denote by int $X, \mathrm{cl} X$, respectively, the interior and the closure of $X$. Consider a $I \times J$-matrix $A$ with $I$ rows and $J$ columns, with entries $A_{i}^{j}(i \in I, j \in J)$, we denote by $A_{i}$ the $i$-th row of $A$ (hence a row vector, i.e., a $(1 \times J)$-matrix, often identified to a vector in $\mathbb{R}^{J}$ when there is no risk of confusion) and $A^{j}$ denotes the $j$-th column of $A$ (hence a column vector, i.e., a $I \times 1$-matrix, which may similarly be identified to a vector in $\mathbb{R}^{I}$ ). If there is no risk of confusion, we will use the same notation for the $I \times J$-matrix $A$ and the associated linear mapping $A: \mathbb{R}^{J} \rightarrow \mathbb{R}^{I}$. We shall denote by $\operatorname{ker} A:=\left\{x \in \mathbb{R}^{J}: A x=0\right\}$ the kernel of $A$, by $\operatorname{Im} A:=\left\{A x: x \in \mathbb{R}^{J}\right\}$ the image of $A$, and by $\operatorname{rank} A$ the $\operatorname{rank}$ of the matrix $A$, that is, the dimension of $\operatorname{Im} A$. We also $\operatorname{denote} \operatorname{ker} A$ by $\{A=0\}$ and we let $\{A \geq 0\}:=\left\{x \in \mathbb{R}^{J}: A x \geq 0\right\}$.
} 
In the exchange economy, there is nonempty finite set $\mathcal{I}:=\{1, \ldots, I\}$ of consumers. Each consumer $i \in \mathcal{I}$ is endowed with a consumption set $X_{i} \subset \mathbb{R}^{L}$, a preference correspondence $P_{i}$, from $\prod_{k \in I} X_{k}$ to $X_{i}$, and an endowment vector $e_{i} \in \mathbb{R}^{L}$. The set $X_{i}$ is the set of her possible consumptions, and for $x \in \prod_{i \in I} X_{i}, P_{i}(x)$ is the set of consumption plans in $X_{i}$ which are strictly preferred to $x_{i}$ by consumer $i$, given the consumption plans $\left(x_{i^{\prime}}\right)_{i^{\prime} \neq i}$ of the other agents. Finally $e_{i}=\left(e_{i}(s)\right)_{\overline{\mathcal{S}}}$ lists the state endowment $e_{i}(s)$ across states, with $e_{i}(0)$ being known with certainty and $e_{i}(s)(s \neq 0)$ being available only if state $s$ prevails at $t=1$. The exchange economy can thus be summarized by $\mathcal{E}=\left(X_{i}, P_{i}, e_{i}\right)_{i \in \mathcal{I}}$.

The financial structure consists of a finite set $\mathcal{J}:=\{1, \ldots, J\}$ of assets. An asset $j$ is a contract which is issued at $t=0$ and promises to deliver the financial payoff $V_{s}^{j}(p)$ at state $s \in \mathcal{S}$ of period $t=1$ if state $s$ prevails (for a given commodity price $p \in \mathbb{R}^{L}$ ). So, the payoff of asset $j$ across tomorrow states is described by the mapping $p \mapsto V^{j}(p):=\left(V_{s}^{j}(p)\right)_{s \in S} \in \mathbb{R}^{S}$. The financial structure is described by the payoff matrix mapping $V: p \mapsto V(p)$, where $V(p)$ is the $S \times J$-matrix, whose columns are the payoffs $V^{j}(p)(1, \ldots, J)$ of the $J$ assets. We denote by $z=\left(z_{j}\right) \in \mathbb{R}^{J}$, the portfolio of some consumer and we will use the standard convention: if $z_{j}>0$ (resp. $z_{j}<0$ ), then $z_{j}$ (resp. $\left|z_{j}\right|$ ) represents the quantity of asset $j$ bought (resp. sold) at period 0 . We assume that portfolios may be constrained, that is, each agent $i$ is endowed with a portfolio set $Z_{i} \subset \mathbb{R}^{J}$ which describes the portfolios that are possible for her. If some agent $i \in \mathcal{I}$ has no constraints on her portfolio choices, then $Z_{i}=\mathbb{R}^{J}$. Throughout this paper we consider portfolio sets that are closed, convex and contain zero for every agent, a framework general enough to cover most of the constraints considered in the literature (see Elsinger and Summer (2001)). The financial structure can be summarized by the list $\mathcal{F}:=\left(V,\left(Z_{i}\right)_{i \in \mathcal{I}}\right)$ and the stochastic financial exchange economy $(\mathcal{E}, \mathcal{F})$ can thus be described by the list

$$
(\mathcal{E}, \mathcal{F}):=\left(\left(X_{i}, P_{i}, e_{i}\right)_{i \in \mathcal{I}} ;\left(V,\left(Z_{i}\right)_{i \in \mathcal{I}}\right)\right)
$$

\subsection{Equilibria and absence of arbitrage opportunities}

Given commodity and asset prices $(p, q) \in \mathbb{R}^{L} \times \mathbb{R}^{J}$, the budget set of consumer $i$ is ${ }^{2}$

$$
\begin{aligned}
B_{\mathcal{F}}^{i}(p, q) & =\left\{\left(x_{i}, z_{i}\right) \in X_{i} \times Z_{i}: \begin{array}{l}
p(0) \cdot x_{i}(0)+q \cdot z_{i} \leq p(0) \cdot e_{i}(0) \\
p(s) \cdot x_{i}(s) \leq p(s) \cdot e_{i}(s)+V_{s}(p) \cdot z_{i}, \quad \forall s \in \mathcal{S}
\end{array}\right\} \\
& =\left\{\left(x_{i}, z_{i}\right) \in X_{i} \times Z_{i}: \quad p \square\left(x_{i}-e_{i}\right) \leq W(p, q) z_{i}\right\},
\end{aligned}
$$

where $W(p, q)$ denotes the total payoff matrix, that is, the $(1+S) \times J$-matrix $\left(\begin{array}{c}-q \\ V(p)\end{array}\right)$.

We now introduce the standard equilibrium notion in this model.

Definition 1. An equilibrium of the economy $(\mathcal{E}, \mathcal{F})$ is a list $(\bar{p}, \bar{q}, \bar{x}, \bar{z}) \in \mathbb{R}^{L} \times \mathbb{R}^{J} \times\left(\mathbb{R}^{L}\right)^{I} \times\left(\mathbb{R}^{J}\right)^{I}$ such that (i) for every $i,\left(\bar{x}_{i}, \bar{z}_{i}\right)$ maximizes the preference $P_{i}$ under the budget constraint, that is

$$
\left(\bar{x}_{i}, \bar{z}_{i}\right) \in B_{\mathcal{F}}^{i}(\bar{p}, \bar{q}) \text { and } B_{\mathcal{F}}^{i}(\bar{p}, \bar{q}) \cap\left(P_{i}(\bar{x}) \times Z_{i}\right)=\emptyset \text {, }
$$

(ii) [Market Clearing] $\sum_{i \in I} \bar{x}_{i}=\sum_{i \in \mathcal{I}} e_{i}$ and $\sum_{i \in \mathcal{I}} \bar{z}_{i}=0$.

\footnotetext{
${ }^{2}$ For every $p=(p(s)), x=(x(s))$ in $\mathbb{R}^{L}$, we denote by $p \square x$ the vector $(p(s) \cdot x(s))_{s \in \overline{\mathcal{S}}}$.
} 
We make the following standard assumptions C1-C6 on the consumption side. We denote by $\mathcal{A}(\mathcal{E})$ the set of attainable allocations of the economy, that is, $\mathcal{A}(\mathcal{E})=\left\{\left(x_{i}\right)_{i \in} \in \prod_{i \in \mathcal{I}} X_{i}: \sum_{i \in \mathcal{I}} x_{i}=\sum_{i \in \mathcal{I}} e_{i}\right\}$.

Consumption Assumption C For every $i \in \mathcal{I}$ and for every $x=\left(x_{i}\right)_{i \in \mathcal{I}} \in \prod_{i} X_{i}$

C1 Consumption Sets: $X_{i}$ is a closed, convex, bounded below subset of $\mathbb{R}^{L}$;

C2 Continuity: The correspondence $P_{i}$, from $\prod_{k \in I} X_{k}$ to $X_{i}$, is lower semicontinuous ${ }^{3}$ with open values in $X_{i}$ (for the relative topology of $X_{i}$ );

C3 Convexity: $P_{i}(x)$ is convex;

C4 Irreflexivity: $x_{i} \notin P_{i}(x)$;

\section{C5 Local Non-Satiation LNS:}

(a) $\forall x \in \mathcal{A}(\mathcal{E}), \forall s \in S, \exists x_{i}^{\prime}(s) \in \mathbb{R}^{\ell},\left(x_{i}^{\prime}(s), x_{i}(-s)\right) \in P_{i}(x){ }^{4}$

(b) $\left.\forall y_{i} \in P_{i}(x)\right],\left(x_{i}, y_{i}\right] \subset P_{i}(x)$;

\section{C6 Consumption Survival CS: $e_{i} \in \operatorname{int} X_{i}$.}

We note that these assumptions are standard in a model with nonordered preferences; the assumptions on $P_{i}$ are satisfied in particular when agents' preferences are represented by utility functions that are continuous, strongly monotonic, and quasi-concave. We now recall that equilibrium asset prices preclude arbitrage opportunities under the above Non-Satiation Assumption. We denote by $\boldsymbol{A Z}$ the asymptotic cone ${ }^{5}$ of a nonempty set $Z \subset \mathbb{R}^{J}$.

Proposition 1. Assume LNS and the convexity of the portfolio sets $Z_{i}(i \in \mathcal{I})$. If $(\bar{p}, \bar{q}, \bar{x}, \bar{z})$ is an equilibrium of the economy $(\mathcal{E}, \mathcal{F})$, then $\bar{q}$ is arbitrage-free at $\bar{p}$, in the sense that

$$
W(\bar{p}, \bar{q})\left(\bigcup_{i} A Z_{i}\right) \cap \mathbb{R}_{+}^{\overline{\mathcal{S}}}=\{0\}
$$

We denote by $Q(\bar{p})$ the set of arbitrage-free asset prices $\bar{q}$ at $\bar{p} \in \mathbb{R}^{L}$.

\footnotetext{
${ }^{3}$ Let $\Phi$ be a correspondence from $X$ to $Y$, that is, $\Phi$ is a mapping from $X$ to $2^{Y}$. Then $\Phi$ is said to be lower semicontinuous (1.s.c.) at $x_{o} \in X$, if for every open set $V \subset Y$ such that $\Phi\left(x_{o}\right) \cap V \neq \emptyset$, there exists an open neighborhood $U$ of $x_{o}$ in $X$ such that $\Phi(x) \cap V \neq \emptyset$ for all every $x \in U$. The correspondence $\Phi$ is said to be 1.s.c. if it is 1.s.c.at every point of $X$. Finally, we denote by $G(\Phi):=\{(x, y) \in X \times Y: y \in \Phi(x)\}$ the graph of $\Phi$.

${ }^{4}$ Given $x_{i} \in X_{i}$ and $s \in \overline{\mathcal{S}}$, we denote $x_{i}(-s):=\left(x_{i}\left(s^{\prime}\right)\right)_{s^{\prime} \neq s}$.

${ }^{5}$ The asymptotic cone of a nonempty subset $Z$ of $\mathbb{R}^{J}$ is the set $A Z:=\left\{\lim _{n} \lambda^{n} z^{n}:\left(\lambda^{n}\right)_{n} \downarrow 0\right.$ and $z^{n} \in Z$ for all $\left.n\right\}$. As a consequence from the definition, one has $A(\mathrm{cl} Z)=A Z$ and we refer to Debreu (1959) for a general reference. When $Z$ is additionally assumed to be convex, then $A Z=0^{+}(\mathrm{cl} Z)$, where $0^{+}(C):=\left\{\zeta \in \mathbb{R}^{J}: \zeta+C \subset C\right\}$ is the recession cone of the convex set $C \subset \mathbb{R}^{J}$ (see Rockafellar (1970)). When $Z$ is convex, the inclusion $0^{+}(Z) \subset A Z$ holds but may be strict when $Z$ is not closed.
} 
Proof. Suppose that for some $i \in I$, there exists a portfolio $\zeta_{i} \in A Z_{i}$ such that $W(\bar{p}, \bar{q}) \zeta_{i}>0$, namely $\left[W(\bar{q}) \zeta_{i}\right](s) \geq 0$, for every $s \in \overline{\mathcal{S}}$, with at least one strict inequality, say for $\bar{s} \in \overline{\mathcal{S}}$. ¿From assumption LNS, there exists $x_{i} \in P_{i}(\bar{x})$ such that $x_{i}(-\bar{s})=\bar{x}_{i}(-\bar{s})$.

For $t>0$ large enough, $\bar{p} \square\left(x_{i}-e_{i}\right) \leq W(\bar{p}, \bar{q})\left(\bar{z}_{i}+t \zeta_{i}\right)$. Since $\bar{z}_{i}+t \zeta_{i} \in Z_{i}$, we get $\left(x_{i}, \bar{z}_{i}+t \zeta_{i}\right) \in B_{\mathcal{F}}^{i}(\bar{p}, \bar{q})$ but since $x_{i} \in P_{i}(\bar{x})$, this contradicts the optimality of $\left(\bar{x}_{i}, \bar{z}_{i}\right)$ in $B_{\mathcal{F}}^{i}(\bar{p}, \bar{q})$.

\subsection{Nominal and numéraire assets}

If the financial structure $\mathcal{F}$ is nominal, the matrix $V(p)$ of financial payoffs does not depend on the commodities price vector $p$ and is denoted $R$.

A numéraire asset is defined as follows. Let us choose a commodity bundle $v \in \mathbb{R}^{\ell}$, a typical example being $v=(0, \ldots, 0,1)$, when the $\ell$-th good is chosen as numéraire. A numéraire asset $j$ is a real asset which delivers the commodity bundle $A_{s}^{j}=R_{s}^{j} v \in \mathbb{R}^{\ell}$ at state $s$ of date $t=1$ if this state $s$ prevails. Thus the payoff at state $s$ is $\left(V_{v}\right)_{s}^{j}(p)=(p(s) \cdot v) R_{s}^{j}$ for the commodity price $p=(p(s)) \in \mathbb{R}^{L}$. For a numéraire financial structure, i.e., all the assets are numéraire assets (for the same commodity bundle $v$ ), we denote $R$ the $S \times J$-matrix with entries $R_{S}^{j}$ and, for $p \in \mathbb{R}^{L}$, we denote $V_{v}(p)$ the associated $S \times J$-payoff-matrix, which has for entries $\left(V_{v}\right)_{s}^{j}(p)=(p(s) \cdot v) R_{s}^{j}$, i.e.

$$
V_{v}(p)=\left(\begin{array}{cccc}
p(1) \cdot v & 0 & \cdots & 0 \\
0 & \ddots & \ddots & \vdots \\
\vdots & \ddots & \ddots & 0 \\
0 & \cdots & 0 & p(S) \cdot v
\end{array}\right) R .
$$

In the nominal case, the set $Q(p)$ of arbitrage-free prices, that is, the set of asset prices $q$ satisfying

$$
\left(\begin{array}{c}
-q \\
R
\end{array}\right)\left(\bigcup_{i} \boldsymbol{A} Z_{i}\right) \cap \mathbb{R}_{+}^{\overline{\mathcal{S}}}=\{0\}
$$

does not depend on the price $p$, hence is simply denoted $Q_{R}$. In the numéraire case, under the Desirability Assumption (made in FN0) below, if $(\bar{p}, \bar{q}, \bar{x}, \bar{z})$ is an equilibrium, then $\bar{p}(s) \cdot v>0$ for all $s \in S$ (see the proof of Lemma 3 in Appendix 4.3) and we notice that, if $\bar{p}(s) \cdot v>0$ for all $s \in S$, then $Q(\bar{p})=Q_{R}$ as defined above by (2.1). Thus, every equilibrium asset price $\bar{q}$ belongs to $Q_{R}$ (by Proposition 1) in the nominal case and in the numéraire case.

Given the financial structure $\mathcal{F}=\left(V,\left(Z_{i}\right)_{i \in \mathcal{I}}\right)$, we denote $Z_{\mathcal{F}}=<\cup_{i \in \mathcal{I}} Z_{i}>$ the linear space where financial activity takes place. We say that $Z \subset \mathbb{R}^{J}$ is a convex polyhedral set if it can be defined by finitely many linear inequalities, i.e., $Z:=\left\{z \in \mathbb{R}^{J}: B z \geq b\right\}$ for some $K \times J$-matrix $B$ and some $b \in \mathbb{R}^{K}$. Clearly this takes into account the case of a set defined by finitely many linear inequalities and/or equality constraints, since each equality constraint can be equivalently replaced by two inequality constraints. We can now present the general assumptions on the financial side:

\section{FN Financial Assumption in the Nominal-Numéraire case:}


FN0: The financial structure $\mathcal{F}$ is either (i) nominal, i.e., $V(p)=R$ is independent of $p$, or (ii) numéraire, i.e., $V(p)=V_{v}(p)$, for every agent $i$, the correspondence $P_{i}$ has an open graph and the commodity bundle $v \in \mathbb{R}^{\ell}$ is desirable at every state $s \in \mathcal{S}$, i.e., for all $x \in \mathcal{A}(\mathcal{E})$, for all $t>0,\left(x_{i}(s)+\right.$ $\left.t v, x_{i}(-s)\right) \in P_{i}(x)$

FN1: For all $i \in \mathcal{I}, Z_{i}$ is a convex polyhedral set, $0 \in Z_{i}$, and $V: \mathbb{R}^{L} \rightarrow \mathbb{R}^{S \times J}$ is continuous;

FN2: Financial Survival $\forall i \in \mathcal{I}, \forall q \in \operatorname{cl} Q_{R} \cap Z_{\mathcal{F}}, q \neq 0, \exists \zeta_{i} \in Z_{i}, q \cdot \zeta_{i}<0$.

Assumption FNO is standard; note however that the open graph assumption and the desirability assumption are slightly stronger than the corresponding ones in $\mathbf{C}$, but these assumptions on $P_{i}$ are still satisfied when $v \in \mathbb{R}_{+}^{\ell} \backslash\{0\}$ and agents' preferences are represented by utility functions that are continuous, strongly monotonic, and quasi-concave. Assumption FN1 considers the class of convex polyhedral portfolio sets of particular interest for economic applications; in particular it takes into account the case of linear equality constraints (portfolio sets which are linear spaces) as considered by Balasko et al. (1990). Sufficient conditions for Assumption FN2 to hold are provided in Section 2.4.

\subsection{Existence of equilibria in the nominal and numéraire case}

We now state our main existence result.

Theorem 1. [Nominal or numéraire assets] Let the economy $(\mathcal{E}, \mathcal{F})$ satisfy assumptions $\mathbf{C}$ and $\mathbf{F N}$, then it admits an equilibrium $(\bar{p}, \bar{q}, \bar{x}, \bar{z})$ such that $\|\bar{p}(0)\|+\|\bar{q}\|=1$ and $\|\bar{p}(s)\|=1$ for $s \in \mathcal{S}$.

The proof of Theorem 1 is given in Section 2.6 as a consequence of an auxiliary result (Theorem 2) stated in the next section. We now give some consequences of Theorem 1. The following Corollary 2 and 3 allow to extend to the case of consumers with nonordered preferences the existence results of Cass (1984), Duffie (1987), and Werner (1985) in the nominal case and Geanakoplos and Polemarchakis (1986) in the numéraire case.

Corollary 1. The financial exchange economy $(\mathcal{E}, \mathcal{F})$ admits an equilibrium under Assumptions $\mathbf{C}$, FN0, FN1 if

- $0 \in$ int $Z_{i}$ for all $i$.

Corollary 2. The financial exchange economy $(\mathcal{E}, \mathcal{F})$ admits an equilibrium under Assumption $\mathbf{C}$ if

- $\mathcal{F}$ consists of nominal assets;

$-Z_{i}=\mathbb{R}^{J}$ for all $i$.

Corollary 3. The financial exchange economy $(\mathcal{E}, \mathcal{F})$ admits an equilibrium under Assumption $\mathbf{C}$ if

- $\mathcal{F}$ consists of numéraire assets and satisfies $\mathbf{F N O}$ (ii);

- $Z_{i}=\mathbb{R}^{J}$ for all $i$. 
The proof of the above corollaries is a consequence of Theorem 1 and of the following proposition, which gives examples under which the Financial Survival Assumption FN2 is satisfied.

Proposition 2. Let $\mathcal{F}=\left(V,\left(Z_{i}\right)_{i}\right)$ be a financial structure, then Assumption $\mathbf{F N 2}$ (and also Assumption $\mathbf{F} 2$ defined hereafter) is satisfied if, either (a) or (b) holds

(a) For every $i \in \mathcal{I}$, one of the following conditions holds:

(i) $Z_{i}=\mathbb{R}^{J}$ (unrestricted participation),

(ii) $Z_{i}=-\underline{z}_{i}+\mathbb{R}_{+}^{J}$, for some $\underline{z}_{i} \in \mathbb{R}_{++}^{J}$ (bounds on short sales for every asset),

(iii) $Z_{i}=\left[-\underline{z}_{i}^{1}+\mathbb{R}_{+}^{J_{1}}\right] \times \mathbb{R}^{J_{2}}$, for some $\underline{z}_{i}^{1} \in \mathbb{R}_{++}^{J_{1}}$, with $J=J_{1}+J_{2}$ (bounds on short sales for some assets),

(iv) $Z_{i}$ is the closed ball $\left\{z \in \mathbb{R}^{J}:\|z\| \leq r\right\}$ for some $r>0$ (bounded portfolios) $)^{6}$,

(v) $0 \in \operatorname{int} Z_{i}$;

(b) For every $i \in \mathcal{I}, Z_{i}=\{0\}$ (pure spot markets).

Proof. Part (a). First notice that each condition (i), (ii), (iii) or (iv) implies (v). Thus we need only show Assumption FN2 holds under Condition (v). Let $q \in\left[\operatorname{cl} Q_{R} \cap Z_{\mathcal{F}}\right] \backslash\{0\}$, and let $i \in I$, then choose $\zeta_{i}=$ $-\varepsilon q /\|q\|$. Then, for $\varepsilon>0$ small enough, $\zeta_{i} \in B(0, \varepsilon) \subset Z_{i}$ (since $0 \in \operatorname{int} Z_{i}$ by Condition $(v)$ ) and $q \cdot \zeta_{i}=$ $-\varepsilon\|q\|<0$.

Part (b). We now prove that Assumption FN2 holds in the case of pure spot markets. Notice that $Z_{\mathcal{F}}=\{0\}$ and $\left[\mathrm{cl} Q_{R} \cap Z_{\mathcal{F}}\right] \backslash\{0\}=\emptyset$, hence FN2 holds.

\subsection{An auxilary existence result}

In this section, we state an auxiliary existence result that will be the key tool to prove Theorem 1 in Section 2.6. This result, also of interest for itself, does not assume neither that the financial structure is nominal or numéraire, nor that the portfolio sets are convex polyhedra. It assumes however the additional Assumption $\mathbf{F 3}$ that is presented below. We posit the following new set of assumptions on the financial structure.

\section{Financial Assumption F:}

F0: The set $\boldsymbol{A}_{\mathcal{F}}(p):=\sum_{i \in \mathcal{I}}\left(A Z_{i} \cap\{V(p) \geq 0\}\right)$ does not depend on $p$ (hence is simply denoted $\boldsymbol{A}_{\mathcal{F}}$ hereafter);

F1: For every $i \in \mathcal{I}, Z_{i}$ is closed, convex, $0 \in Z_{i}$, and $V: \mathbb{R}^{L} \rightarrow \mathbb{R}^{S \times J}$ is continuous;

F2: Financial Survival $\forall i \in \mathcal{I}, \forall p \in \mathbb{R}^{L}, p(0)=0, \forall q \in \operatorname{cl} Q(p) \cap Z_{\mathcal{F}}, q \neq 0, \exists \zeta_{i} \in Z_{i}, q \cdot \zeta_{i}<0$;

F3: The set $\boldsymbol{A}_{\mathcal{F}}$ is pointed, i.e., $\boldsymbol{A}_{\mathcal{F}} \cap-\boldsymbol{A}_{\mathcal{F}}=\{0\}$.

Assumptions F0 and F1 weaken the previous assumptions FN0 and FN1, respectively, and F2 adapts the previous Survival Assumption FN2 to the more general case considered here. Sufficient conditions for Assumptions F0 and $\mathbf{F 3}$ to hold are given at the end of this section.

We now state our second existence result.

\footnotetext{
${ }^{6}$ The result is true for any norm $\|\cdot\|$ of $\mathbb{R}^{J}$ and the set $Z_{i}$ will be polyhedral if we take for example the norm $\|z\|_{\infty}:=\max \left\{\left|z_{j}\right|:\right.$ $j=1 \ldots, J\}$.
} 
Theorem 2. Let the economy $(\mathcal{E}, \mathcal{F})$ satisfy assumptions $\mathbf{C}$ and $\mathbf{F}$, then it admits an equilibrium $(\bar{p}, \bar{q}, \bar{x}, \bar{z})$ such that $\|\bar{p}(0)\|+\|\bar{q}\|=1$ and $\|\bar{p}(s)\|=1$ for $s \in \mathcal{S}$.

The proof of Theorem 2 is given in Section 3.

We can now give two consequences of Theorem 2 which extend the results by Radner (1972) and the framework of Siconolfi (1989) to the case of agents with nonordered preferences. Note however that the Survival Assumption F2 is not directly comparable to the one used by Siconolfi (1989) and it is the purpose of further work to show the relationship between his existence result and Theorem 2.

Corollary 4. The economy $(\mathcal{E}, \mathcal{F})$ admits an equilibrium under assumptions $\mathbf{C}, \mathbf{F 1}$ in each of the following cases:

(i) (Radner (1972)) For every $i \in I, Z_{i}$ is the closed ball $B_{J}\left(0, r_{i}\right)$, for some $r_{i}>0$.

(ii) (Siconolfi (1989)) $\mathcal{F}$ consists of nominal assets, $\mathbf{F} 2$ holds and $\boldsymbol{A} Z_{i} \cap \operatorname{ker} R=\{0\}$ for every $i \in \mathcal{I}$.

The proof of the corollary is a consequence of Theorem 2, Proposition 2, and Remarks 1, and 2, which give sufficient conditions for Assumptions F2, F0, and F3 to hold.

Remark 1. Assumption F0 is satisfied when

- the financial structure is nominal, i.e., $V(p)$ does not depend on $p$;

- the set $\{V(p) \geq 0\}$ does not depend on $p$;

- for every $i, Z_{i}$ is bounded (Radner (1972)) since $A Z_{i}=\{0\}$;

- the set $\operatorname{cl} Q(p)$ does not depend on $p$ (under Assumption F3, by Lemma 4 below);

- the case of numéraire assets is treated in Section 2.6.

Remark 2. Assumption F3 is satisfied when

- $\operatorname{ker} V=\{0\}$ (No redundant assets);

- for every $i, Z_{i}$ is bounded (Radner (1972));

- for every $i, \operatorname{ker} V \cap A Z_{i}=\{0\}$ (Siconolfi (1989)).

Moreover, Assumption $\mathbf{F 3}$ is equivalent to the assumption that $V$ has no redundant assets when there are no restrictions on portfolio trades $\left(Z_{i}=\mathbb{R}^{J}\right.$ for all $\left.i\right)$. In the case of nominal assets, it coincides with Siconolfi (1989)'s assumption when the $Z_{i}$ 's are linear subspaces.

Assumption F3 is crucial in the proof of Theorem 2. Together with F0, it enables us to look for equilibrium portfolios in an appropriate bounded set, creating thereby a suitable environment for the application of a fixed point theorem. In this regard, Assumption F3 and Siconolfi (1989)'s assumption serve the same purpose, but the weaker Assumption $\mathbf{F 3}$ is needed if we want to treat the case of linear inequalities instead of linear equalities. See Section 2.6 for more detail.

Remark 3. We can choose the equilibrium asset price $\bar{q}$ to be in $Q(\bar{p}) \cap Z_{\mathcal{F}}$. Indeed, let $(\bar{p}, \bar{q}, \bar{x}, \bar{z})$ be an equilibrium of $(\mathcal{E}, \mathcal{F})$ and let $q^{*}=\operatorname{proj}_{Z_{\mathcal{F}}} \bar{q}$ then $\left(\bar{p}, q^{*}, \bar{x}, \bar{z}\right)$ is an equilibrium of $(\mathcal{E}, \mathcal{F})$ since for every $i \in \mathcal{I}$, and for every $z_{i} \in Z_{i}$, one has $q^{*} \cdot z_{i}=\bar{q} \cdot z_{i}$. Moreover, $q^{*} \in Q(\bar{p})$ (under LNS by Proposition 1 ). 


\subsection{Proof of Theorem 1}

The proof of Theorem 1 is given hereafter, first in the case of nominal assets and then in the case of numéraire assets. Consider an economy $(\mathcal{E}, \mathcal{F})$ satisfying Assumptions $\mathbf{C}$ and $\mathbf{F N}$.

In the case of nominal assets with no restrictions on portfolio trades, there is a priori no loss of generality in assuming that the payoff matrix $V$ has no redundant assets, that is, $\operatorname{rank} V=J$ or equivalently $\operatorname{ker} V=\{0\}$. Indeed, otherwise by deleting the redundant columns we obtain a new financial economy whose equilibria yield equilibria in the original one. Hereafter, we show how to transform the financial structure to obtain a reduced financial structure, i.e., satisfying Assumption F3, which supersedes the standard assumption of no redundant assets, while keeping the correspondence between the equilibria. Existence of equilibrium then follows from Theorem 2. This analysis coincides with the one in Balasko et al. (1990) when portfolio sets are defined by linear equality constraints and assets are nominal (since the transformed financial structure they obtain satisfies Siconolfi (1989)'s assumption which is equivalent to F3 in the framework of linear equality constraints), and extends it to the case of linear inequality constraints with nominal and numéraire assets.

We modify the financial structure $\mathcal{F}$ by considering the reduced financial structure $\mathcal{F}_{\pi}$ which has the same payoff matrix as $\mathcal{F}$ and the portfolio sets $\pi Z_{i}(i \in \mathcal{I})$ where $\pi$ is the orthogonal projection mapping ${ }^{7}$ on the orthogonal space to $\boldsymbol{L}_{\mathcal{F}}:=\boldsymbol{A}_{\mathcal{F}} \cap-\boldsymbol{A}_{\mathcal{F}}$. We recall that $Z_{\mathcal{F}}:=<\cup_{i} Z_{i}>, Z_{\mathcal{F}_{\pi}}:=<\cup_{i} \pi Z_{i}>$ and the definition of $\mathcal{F}_{\pi}$ can be summarized by

$$
\begin{gathered}
\mathcal{F}_{\pi}=\left(V(p),\left(\pi Z_{i}\right)_{i}\right), \\
\text { where } \pi=\operatorname{proj}_{\left(\boldsymbol{L}_{\mathcal{F}}\right)^{\perp}}, \boldsymbol{L}_{\mathcal{F}}:=\boldsymbol{A}_{\mathcal{F}} \cap-\boldsymbol{A}_{\mathcal{F}} \text {, and } \boldsymbol{A}_{\mathcal{F}}:=\sum_{i \in \mathcal{I}}\left(A Z_{i} \cap\{V(p) \geq 0\}\right) \subset Z_{\mathcal{F}}
\end{gathered}
$$

\subsubsection{The nominal case}

This section considers the case of a financial structure $\mathcal{F}=\left(V,\left(Z_{i}\right)_{i}\right)$ with nominal assets satisfying the Assumption FN, and in fact the more general case of a financial structure satisfying Assumptions F0, FN1, and F2. This more general framework will be needed in Section 2.6.2 to treat the case of numéraire assets.

Step 1. The first step is summarized by Lemma 1, the proof of which is given in Appendix 4.1.

Lemma 1. If $\mathcal{F}=\left(V,\left(Z_{i}\right)_{i}\right)$ satisfies Assumptions $\mathbf{F 0}, \mathbf{F N 1}$, and $\mathbf{F} 2$, then $\mathcal{F}_{\pi}$ satisfies Assumptions $\mathbf{F 0}-\mathbf{F} 2$, together with Assumption $\mathbf{F 3}$, that is, $\boldsymbol{A}_{\mathcal{F}_{\pi}} \cap-\boldsymbol{A}_{\mathcal{F}_{\pi}}=\{0\}$.

Step 2. From Theorem 2 and Step 1, the economy $\left(\mathcal{E}, \mathcal{F}_{\pi}\right)$ admits an equilibrium $(\bar{p}, \bar{q}, \bar{x}, \bar{z})$.

Step 3. There exists an equilibrium $\left(p^{*}, q^{*}, x^{*}, z^{*}\right)$ of $(\mathcal{E}, \mathcal{F})$ as a consequence of the following lemma, the proof of which is given in the Appendix 4.2.

\footnotetext{
${ }^{7}$ When $L$ is a subset of $\mathbb{R}^{J}$, we define the polar set of $L$ by $L^{o}:=\left\{z \in \mathbb{R}^{J}: z \cdot \xi \leq 0\right.$ for all $\left.\xi \in L\right\}$ and the orthogonal set to $L$ by $L^{\perp}:=\left\{z \in \mathbb{R}^{J}: z \cdot \xi=0\right.$ for all $\left.\xi \in L\right\}$. When $L$ is a linear space and $\varphi \in \mathbb{R}^{J}$, we denote by $\operatorname{proj}_{L} \varphi\left(\right.$ resp. proj $\left.L_{L^{\perp}} \varphi\right)$ the orthogonal projection of $\varphi$ on $L$ (resp. on $L^{\perp}$ ), that is, the unique $\alpha \in L$ (resp. $\beta \in L^{\perp}$ ) such that $\varphi-\alpha \in L^{\perp}$ (resp. $\varphi-\beta \in L$ ).
} 
Lemma 2. Assume LNS, and let $(\bar{p}, \bar{q}, \bar{x}, \bar{z})$ be an equilibrium of $\left(\mathcal{E}, \mathcal{F}_{\pi}\right)$. Then there exists $z^{*} \in \prod_{i} Z_{i}$ such that $\left(\bar{p}, \pi \bar{q}, \bar{x}, z^{*}\right)$ is an equilibrium of $(\mathcal{E}, \mathcal{F})$.

To end the proof of Theorem 1 we need to check that the equilibrium found in Lemma 2 can be chosen so that $\|\bar{p}(0)\|+\|\pi \bar{q}\|=1$ and $\|\bar{p}(s)\|=1$ for $s \in \mathcal{S}$. Recall that from Remark 3 we can choose the equilibrium asset price vector in $\left(\mathcal{E}, \mathcal{F}_{\pi}\right)$, that is $\bar{q}$, to be in $Q_{\mathcal{F}_{\pi}}(\bar{p}) \cap Z_{\mathcal{F}_{\pi}} \subset Z_{\mathcal{F}_{\pi}} \subset \operatorname{Im} \pi$. Hence $\pi \bar{q}=\bar{q}$ and $\|\bar{p}(0)\|+\|\pi \bar{q}\|=\|\bar{p}(0)\|+\|\bar{q}\|=1$. The fact $\|\bar{p}(s)\|=1$ for $s \in \mathcal{S}$ is immediate since we are not changing the equilibrium commodity prices when we go from an equilibrium in $\left(\mathcal{E}, \mathcal{F}_{\pi}\right)$ to an equilibrium in $(\mathcal{E}, \mathcal{F})$.

\subsubsection{The numéraire case}

Consider a financial economy with numéraire assets $(\mathcal{E}, \mathcal{F})$ satisfying Assumptions $\mathbf{F N}$ (the part (ii) with numéraire assets). The proof of Theorem 1 consists in applying the Steps 1-3 of the previous section to a modified financial economy $\left(\mathcal{E}, \mathcal{F}^{\varepsilon}\right.$ ) (for $\varepsilon>0$ small enough), chosen so that $(i)$ it satisfies Assumptions F0, FN1, and $\mathbf{F} 2$ and $(i i)$ the equilibria of $\left(\mathcal{E}, \mathcal{F}^{\varepsilon}\right)$ are also equilibria of the original financial economy $(\mathcal{E}, \mathcal{F})$.

Step 0. We define the modified financial structure $\mathcal{F}^{\varepsilon}=\left(V^{\varepsilon},\left(Z_{i}\right)_{i}\right)$ for $\varepsilon>0$, by taking the same portfolio sets $Z_{i}$ as for $\mathcal{F}$ and defining the modified payoff matrix $V^{\varepsilon}$, by

$$
V^{\varepsilon}(p)=\left(\begin{array}{cccc}
\max \{\varepsilon, p(1) \cdot v\} & 0 & \cdots & 0 \\
0 & \ddots & \ddots & \vdots \\
\vdots & \ddots & \ddots & 0 \\
0 & \cdots & 0 & \max \{\varepsilon, p(S) \cdot v\}
\end{array}\right) R .
$$

The financial exchange economy $\left(\mathcal{E}, \mathcal{F}^{\varepsilon}\right)$ satisfies Assumptions F0, FN1, and F2 whenever the economy $(\mathcal{E}, \mathcal{F})$ satisfies Assumptions FN0-FN2. Indeed, $\left\{V^{\varepsilon}(p) \geq 0\right\}=\{R \geq 0\}$ for every $p \in \mathbb{R}^{L}$, hence $V^{\varepsilon}$ satisfies Assumption Fo. Assumptions F1 and FN1 are obviously satisfied, and assumption F2 is a consequence of FN2 and the fact that $Q_{\mathcal{F} \varepsilon}(p)=Q_{R}$ for every $p$. The relationship between the equilibria of the economies $\left(\mathcal{E}, \mathcal{F}^{\varepsilon}\right)$ and $(\mathcal{E}, \mathcal{F})$ is then given by the following lemma, the proof of which is given in Appendix 4.3.

Lemma 3. For $\varepsilon>0$ small enough, every equilibrium $(\bar{p}, \bar{q}, \bar{x}, \bar{z})$ of $\left(\mathcal{E}, \mathcal{F}^{\varepsilon}\right)$ such that $\|\bar{p}(s)\|=1$ for $s \in \mathcal{S}$ is an equilibrium of the economy $(\mathcal{E}, \mathcal{F})$.

\section{Proof of Theorem 2}

We first state a lemma summarizing several properties that will be used hereafter. We recall that

$$
\boldsymbol{A}_{\mathcal{F}}(p):=\sum_{i \in \mathcal{I}}\left(A Z_{i} \cap\{V(p) \geq 0\}\right) \subset Z_{\mathcal{F}} .
$$


Lemma 4. Under Assumptions $\mathbf{F 1}$ and $\mathbf{F 3}$, for every $p \in \mathbb{R}^{L}$ :

(a) The set $Q(p)$ is a convex cone with vertex 0 .

(b) $\left(\operatorname{cl} Q(p) \cap Z_{\mathcal{F}}\right)^{o}=-\boldsymbol{A}_{\mathcal{F}}(p)+\left(Z_{\mathcal{F}}\right)^{\perp}$. Hence $\boldsymbol{A}_{\mathcal{F}}(p) \subset Z_{\mathcal{F}}$ is closed

(c) If we additionally assume $\mathbf{F 0}$, then for all $v=\left(v_{i}\right)_{i} \in\left(\mathbb{R}^{S}\right)^{I}$ the set $K_{v}$ is bounded, where

$$
K_{v}:=\left\{\left(z_{1}, \cdots, z_{I}, p\right) \in\left(\prod_{i} Z_{i}\right) \times B_{L}(0,1): \forall i, V(p) z_{i} \geq v_{i},-\sum_{i \in \mathcal{I}} z_{i} \in \boldsymbol{A}_{\mathcal{F}}\right\} .
$$

The proof of Lemma 4 is given in the Appendix 4.4.

\subsection{Truncating the economy}

We denote by $\widehat{X}_{i}$ the projection of the set of attainable allocations $\mathcal{A}(\mathcal{E})$ on $X_{i}$. Since $\mathcal{A}(\mathcal{E})$ is bounded (by Assumption C1), the sets $\widehat{X}_{i}$ are also bounded for every $i \in \mathcal{I}$. Consequently, one can choose $r_{1}>0$ large enough such that

$$
\widehat{X}_{i} \subset \operatorname{int} B_{L}\left(0, r_{1}\right) \text { for every } i \in \mathcal{I},
$$

For $i \in \mathcal{I}$, let $\underline{v}_{i}=\left(\underline{v}_{i}(s)\right) \in \mathbb{R}^{S}$, where

$$
\underline{v}_{i}(s)=-1+\min \left\{p(s) \cdot\left(x_{i}(s)-e_{i}(s)\right): p(s) \in B_{\ell}(0,1), x_{i} \in B_{L}\left(0, r_{1}\right)\right\} \quad(s \in \mathcal{S}),
$$

which is well defined from the compactness of the closed balls $B_{\ell}(0,1)$ and $B_{L}\left(0, r_{1}\right)$. We denote by $\widehat{Z}_{i}$ the projection of $K_{\underline{v}}$ on $Z_{i}$ and the sets $\widehat{Z}_{i}$ are bounded for every $i \in \mathcal{I}$, since $K_{\underline{v}}$ is bounded (by Lemma 4). Consequently, one can choose $r_{2}>0$ large enough such that

$$
\widehat{Z}_{i} \subset \operatorname{int} B_{J}\left(0, r_{2}\right) \text { for every } i \in \mathcal{I} \text {. }
$$

We let $r=\left(r_{1}, r_{2}\right)$ and for every $i \in \mathcal{I}$,

$$
X_{i}^{r}=X_{i} \cap B_{L}\left(0, r_{1}\right), \quad P_{i}^{r}(x)=P_{i}(x) \cap \operatorname{int} B_{L}\left(0, r_{1}\right), \quad Z_{i}^{r}=Z_{i} \cap B_{J}\left(0, r_{2}\right) .
$$

We define the truncated financial economy $\left(\mathcal{E}^{r}, \mathcal{F}^{r}\right)$, which has $X_{i}^{r}$, for consumption sets, $P_{i}^{r}$, for preference correspondences, $Z_{i}^{r}$ for portfolio sets. The endowments of consumers and the payoff matrix are the same as for the economy $(\mathcal{E}, \mathcal{F})$. To summarize, we let

$$
\left(\mathcal{E}^{r}, \mathcal{F}^{r}\right):=\left(\left(X_{i}^{r}, P_{i}^{r}, e_{i}\right)_{i \in \mathcal{I}},\left(V,\left(Z_{i}^{r}\right)_{i \in \mathcal{I}}\right)\right)
$$

\subsection{The reaction correspondences and the fixed-point argument}

We define the set of admissible prices for commodities and assets

$$
\Pi=\left\{(p, q) \in \mathbb{R}^{L} \times \mathbb{R}^{J}:\|p(0)\|+\|q\| \leq 1, \forall s \in \mathcal{S},\|p(s)\| \leq 1, \text { and } q \in \operatorname{cl} Q(p) \cap Z_{\mathcal{F}}\right\},
$$

and, following Bergstrom (1976), the "modified" budget sets of consumer $i$, for $(p, q) \in \Pi$ :

$$
\begin{aligned}
& B_{i}^{r \varepsilon}(p, q)=\left\{\left(x_{i}, z_{i}\right) \in X_{i}^{r} \times Z_{i}^{r}: p \square\left(x_{i}-e_{i}\right) \leq W(p, q) z_{i}+\varepsilon(p, q)\right\}, \\
& \breve{B}_{i}^{r \varepsilon}(p, q)=\left\{\left(x_{i}, z_{i}\right) \in X_{i}^{r} \times Z_{i}^{r}: p \square\left(x_{i}-e_{i}\right) \ll W(p, q) z_{i}+\varepsilon(p, q)\right\},
\end{aligned}
$$


where $\varepsilon(p, q)=\left(\varepsilon_{s}(p, q)\right)_{s \in \overline{\mathcal{S}}} \in[0,1]^{1+S}$ is defined, for $(p, q) \in \Pi$, by

$$
\varepsilon_{s}(p, q)=\left\{\begin{array}{lr}
1-\|p(0)\|-\|q\| & \text { for } s=0 \\
1-\|p(s)\| & \text { for } s \in \mathcal{S}
\end{array}\right.
$$

Claim 3.1. For all $(p, q) \in \Pi, \breve{B}_{i}^{r \varepsilon}(p, q) \neq \emptyset$ and $B_{i}^{r \varepsilon}(p, q)=\operatorname{cl}_{i}^{r \varepsilon}(p, q)$.

Proof. We first notice that $e_{i} \in \operatorname{int} X_{i}^{r}$ for every $i \in \mathcal{I}$; indeed, this is a consequence of the facts that $e_{i} \in \widehat{X}_{i} \subset X_{i} \cap \operatorname{int} B_{L}\left(0, r_{1}\right)$ and $e_{i} \in \operatorname{int} X_{i}$ (Survival Assumption C6). Let $(p, q) \in \Pi$. Since $e_{i} \in \operatorname{int} X_{i}^{r}$, for $t>0$ small enough, $x_{i}^{r}:=e_{i}-t p \in X_{i}^{r}$. Hence, $p \square\left(x_{i}^{r}-e_{i}\right)=-t p \square p \leq 0$, and

$$
\begin{aligned}
& p(0) \cdot\left(x_{i}^{r}(0)-e_{i}(0)\right)-\varepsilon_{0}(p, q)=-t\|p(0)\|^{2}-1+\|p(0)\|+\|q\|<0 \text { if }[p(0) \neq 0 \text { or } q=0], \\
& p(s) \cdot\left(x_{i}^{r}(s)-e_{i}(s)\right)-\varepsilon_{s}(p, q)=-t\|p(s)\|^{2}-1+\|p(s)\|<0 \text { for all } s=1, \ldots, S .
\end{aligned}
$$

Consequently, if $p(0) \neq 0$ or $q=0$, then $\left(x_{i}^{r}, 0\right) \in \breve{B}_{i}^{r \varepsilon}(p, q)$. Assume now that $p(0)=0$ and $q \neq 0$, then there exists $z_{i} \in Z_{i}$ such that $q \cdot z_{i}<0$ (by Assumption F2). We can choose $\lambda>0$ small enough $\left(\lambda\left\|z_{i}\right\| \leq r_{2}\right)$ so that $z_{i}^{r}:=\lambda z_{i} \in Z_{i}^{r}:=Z_{i} \cap B\left(0, r_{2}\right)$ (since $z_{i}^{r} \in B\left(0, r_{2}\right)$, and $z_{i}^{r} \in Z_{i}$ recalling that $z_{i} \in Z_{i}$, $0 \in Z_{i}$ and $Z_{i}$ is convex) and $q \cdot z_{i}^{r}<0$. Again, from above, we can choose $\lambda>0$ small enough so that $p(s) \cdot\left(x_{i}^{r}(s)-e_{i}(s)\right)-\varepsilon_{s}(p, q)<V_{s}(p) \cdot z_{i}^{r}$ for all $s \in \mathcal{S}$. Thus, $\left(x_{i}^{r}, z_{i}^{r}\right) \in \breve{B}_{i}^{r \varepsilon}(p, q) \neq \emptyset$.

We now prove the equality $B_{i}^{r \varepsilon}(p, q)=\operatorname{cl} \breve{B}_{i}^{r \varepsilon}(p, q)$. The first inclusion $\operatorname{cl} \breve{B}_{i}^{r \varepsilon}(p, q) \subset B_{i}^{r \varepsilon}(p, q)$ is immediate. Conversely, let $\left(x_{i}, z_{i}\right) \in B_{i}^{r \varepsilon}(p, q)$ and let $\left(\breve{x}_{i}, \breve{z}_{i}\right) \in \breve{B}_{i}^{r \varepsilon}(p, q) \neq \emptyset$, then $\left(x_{i}, z_{i}\right)=\lim _{t \rightarrow 0}\left(x_{i}^{t}, z_{i}^{t}\right) \in$ $\operatorname{cl} \breve{B}_{i}^{r \varepsilon}(p, q)$ taking $\left(x_{i}^{t}, z_{i}^{t}\right):=(1-t)\left(x_{i}, z_{i}\right)+t\left(\breve{x}_{i}, \breve{z}_{i}\right) \in \breve{B}_{i}^{r \varepsilon}(p, q)$ for all $t \in[0,1]$.

We now introduce an additional agent and, as in (Gale and Mas-Colell $(1975,1979))$, we set the following reaction correspondences defined on $\Pi \times \prod_{i \in I} X_{i}^{r} \times Z_{i}^{r}$

$$
\begin{gathered}
\Phi_{i}(p, q, x, z)=\left\{\begin{array}{l}
B_{i}^{r \varepsilon}(p, q) \text { if }\left(x_{i}, z_{i}\right) \notin B_{i}^{r \varepsilon}(p, q) \\
\breve{B}_{i}^{r \varepsilon}(p, q) \cap\left(P_{i}^{r}(x) \times Z_{i}^{r}\right) \text { if }\left(x_{i}, z_{i}\right) \in B_{i}^{r \varepsilon}(p, q),
\end{array}\right. \\
\Phi_{0}(p, q, x, z)=\left\{\left(p^{\prime}, q^{\prime}\right) \in \Pi:\left(p^{\prime}-p\right) \cdot \sum_{i \in I}\left(x_{i}-e_{i}\right)+\left(q^{\prime}-q\right) \cdot \sum_{i \in I} z_{i}>0\right\} .
\end{gathered}
$$

The main properties of the correspondences $\Phi_{i}$ are stated in the following lemma, the proof of which is given in Appendix 4.5.

Lemma 5. For all $i \in\{0\} \cup \mathcal{I}$, the correspondence $\Phi_{i}$ is lower semicontinuous with convex values on $\Pi \times \prod_{i \in \mathcal{I}} X_{i}^{r} \times Z_{i}^{r}$.

The proof of Theorem 2 relies on the following theorem due to Gale and Mas-Colell (1975).

Theorem 3. Let $I_{o}$ be a finite set, let $C_{i}\left(i \in I_{o}\right)$ be a nonempty, compact, convex subset of some Euclidean space, let $C=\prod_{i \in \mathcal{I}_{0}} C_{i}$ and let $\Phi_{i}\left(i \in \mathcal{I}_{o}\right)$ be a correspondence from $C$ to $C_{i}$, which is lower semicontinuous and convex-valued. Then, there exists $c^{*}=\left(c_{i}^{*}\right)_{i} \in C$ such that, for every $i \in \mathcal{I}_{0}$ [either $c_{i}^{*} \in \Phi_{i}\left(c^{*}\right)$ or $\left.\Phi_{i}\left(c^{*}\right)=\emptyset\right]$ 
We apply Theorem 3 to the sets $\mathcal{I}_{o}=\{0\} \cup \mathcal{I}, C_{0}=\Pi, C_{i}=X_{i}^{r} \times Z_{i}^{r}(i \in \mathcal{I})$, and to the correspondences $\Phi_{i}$ $\left(i \in \mathcal{I}_{o}\right.$ ) defined above. We check that the assumptions of Theorem 3 are fulfilled. The set $\Pi$ is convex since the set $\operatorname{cl} Q(p) \cap Z_{\mathcal{F}}$ is convex and does not depend on $p$ (by Assumption $\mathbf{F 0}$ and Lemma 4). Moreover $\Pi$ is obviously compact, and nonempty (since $(0,0) \in \Pi$ ). For every $i \in \mathcal{I}$, the set $X_{i}^{r} \times Z_{i}^{r}$ is clearly compact, convex, and nonempty (since it contains $\left(e_{i}, 0\right)$ ). Finally, for every $i \in \mathcal{I}_{o}$, the correspondence $\Phi_{i}$ is lower semicontinuous and convex-valued by Lemma 5.

It follows from Theorem 3 that there exists $(\bar{p}, \bar{q}, \bar{x}, \bar{z}) \in \Pi \times \prod_{i \in I}\left(X_{i}^{r} \times Z_{i}^{r}\right)$ such that for all $i \in \mathcal{I}$, either $\Phi_{i}(\bar{p}, \bar{q}, \bar{x}, \bar{z})=\emptyset$ or $\left(\bar{x}_{i}, \bar{z}_{i}\right) \in \Phi_{i}(\bar{p}, \bar{q}, \bar{x}, \bar{z})$, and for $i=0$, either $\Phi_{0}(\bar{p}, \bar{q}, \bar{x}, \bar{z})=\emptyset$ or $(\bar{p}, \bar{q}) \in \Phi_{0}(\bar{p}, \bar{q}, \bar{x}, \bar{z})$. Remark that, by construction, $(\bar{p}, \bar{q}) \notin \Phi_{0}(\bar{p}, \bar{q}, \bar{x}, \bar{z})$, hence $\Phi_{0}(\bar{p}, \bar{q}, \bar{x}, \bar{z})=\emptyset$ or equivalently

$$
p \cdot \sum_{i \in \mathcal{I}}\left(\bar{x}_{i}-e_{i}\right)+q \cdot \sum_{i \in \mathcal{I}} \bar{z}_{i} \leq \bar{p} \cdot \sum_{i \in \mathcal{I}}\left(\bar{x}_{i}-e_{i}\right)+\bar{q} \cdot \sum_{i \in \mathcal{I}} \bar{z}_{i} \quad \text { for all }(p, q) \in \Pi .
$$

Moreover, for every $i \in \mathcal{I},\left(\bar{x}_{i}, \bar{z}_{i}\right) \in B_{i}^{r \varepsilon}(\bar{p}, \bar{q})$; indeed if $\left(\bar{x}_{i}, \bar{z}_{i}\right) \notin B_{i}^{r \varepsilon}(\bar{p}, \bar{q})$, then $\left(\bar{x}_{i}, \bar{z}_{i}\right) \notin \Phi_{i}(\bar{p}, \bar{q}, \bar{x}, \bar{z})=$ $B_{i}^{r \varepsilon}(\bar{p}, \bar{q})$ and $\Phi_{i}(\bar{p}, \bar{q}, \bar{x}, \bar{z}) \neq \emptyset$ (since it contains $\left(e_{i}, 0\right)$ ), a contradiction. Consequently, for all $i \in \mathcal{I}$, either $\breve{B}_{i}^{r \varepsilon}(\bar{p}, \bar{q}) \cap\left(P_{i}^{r}(\bar{x}) \times Z_{i}^{r}\right)=\emptyset$ or $\left(\bar{x}_{i}, \bar{z}_{i}\right) \in \breve{B}_{i}^{r \varepsilon}(\bar{p}, \bar{q}) \cap\left(P_{i}^{r}(\bar{x}) \times Z_{i}^{r}\right)$ and the second condition cannot hold from the irreflexivity of $P_{i}^{r}$ (by Assumption $\mathbf{C}$ ). Thus we can conclude that

$$
\text { for all } i \in \mathcal{I},\left(\bar{x}_{i}, \bar{z}_{i}\right) \in B_{i}^{r \varepsilon}(\bar{p}, \bar{q}) \text { and } \breve{B}_{i}^{r \varepsilon}(\bar{p}, \bar{q}) \cap\left(P_{i}^{r}(\bar{x}) \times Z_{i}^{r}\right)=\emptyset \text {. }
$$

\subsection{Checking the market clearing conditions}

Since the Market Clearing Condition $\sum_{i \in I} \bar{z}_{i}=0$ may not be satisfied by the portfolios $\bar{z}=\left(\bar{z}_{i}\right)_{i}$, the purpose of the next claim is to define new portfolios $\overline{\bar{z}}_{i}(i \in \mathcal{I})$ that will satisfy the Market Clearing Condition $\sum_{i \in I} \overline{\bar{z}}_{i}=0$ and will also be admissible, i.e., $\overline{\bar{z}}_{i} \in \widehat{Z}_{i} \subset Z_{i}^{r}$ for all $i$.

Claim 3.2. (a) For every $i, V(\bar{p}) \bar{z}_{i} \geq \underline{v}_{i}, \sum_{i \in I} \bar{z}_{i} \in-\boldsymbol{A}_{\mathcal{F}}$, and $\bar{q} \cdot \sum_{i \in I} \bar{z}_{i}=0$.

We let $\overline{\bar{z}}_{i}=\bar{z}_{i}+\zeta_{i}$, for some $\zeta_{i} \in A Z_{i} \cap\{V(\bar{p}) \geq 0\}(i \in \mathcal{I})$ such that $\sum_{i \in \mathcal{I}} \bar{z}_{i}=-\sum_{i \in \mathcal{I}} \zeta_{i}$.

(b) Then $\sum_{i \in I} \overline{\bar{z}}_{i}=0$, for every $i, \overline{\bar{z}}_{i} \in \widehat{Z}_{i} \subset Z_{i}^{r}, \bar{q} \cdot \overline{\bar{z}}_{i}=\bar{q} \cdot \bar{z}_{i}, V(\bar{p}) \overline{\bar{z}}_{i} \geq V(\bar{p}) \bar{z}_{i}$ and $\left(\bar{x}_{i}, \overline{\bar{z}}_{i}\right) \in B_{i}^{r \varepsilon}(\bar{p}, \bar{q})$.

Proof. Part $(a)$. First, $V(\bar{p}) \bar{z}_{i} \geq \underline{v}_{i}$ follows from the definition of $\underline{v}_{i}$ (in (3.1)) and $\left(\bar{x}_{i}, \bar{z}_{i}\right) \in B_{i}^{r \varepsilon}(\bar{p}, \bar{q})$ (by (3.3)).

Second, we show that $\sum_{i \in I} \bar{z}_{i} \in\left(\operatorname{cl} Q(\bar{p}) \cap Z_{\mathcal{F}}\right)^{o}$. If this does not hold, then there exists $q^{\prime} \in \operatorname{cl} Q(\bar{p}) \cap Z_{\mathcal{F}}$ such that $q^{\prime} \cdot\left(\sum_{i \in I} \bar{z}_{i}\right)>0$. Without any loss of generality, we can assume that $q^{\prime} \in B_{J}(0,1)$. From (3.2) (taking $(p, q) \in \Pi$ defined by $p(0)=0, p(s)=\bar{p}(s)$ for $s \neq 0$ and $\left.q=q^{\prime}\right)$ we have

$$
0<q^{\prime} \cdot \sum_{i \in \mathcal{I}} \bar{z}_{i} \leq \bar{p}(0) \cdot \sum_{i \in \mathcal{I}}\left(\bar{x}_{i}(0)-e_{i}(0)\right)+\bar{q} \cdot \sum_{i \in \mathcal{I}} \bar{z}_{i}
$$

Since $\left(\bar{x}_{i}, \bar{z}_{i}\right) \in B_{i}^{r \varepsilon}(\bar{p}, \bar{q})$ (by (3.3)) we deduce that

$$
\bar{p}(0) \cdot\left(\bar{x}_{i}(0)-e_{i}(0)\right)+\bar{q} \cdot \bar{z}_{i} \leq \varepsilon_{0}(\bar{p}, \bar{q}):=1-\|\bar{p}(0)\|-\|\bar{q}\| \text { for all } i \in \mathcal{I} .
$$


Summing up over $i$ we get

$$
\bar{p}(0) \cdot \sum_{i \in \mathcal{I}}\left(\bar{x}_{i}(0)-e_{i}(0)\right)+\bar{q} \cdot \sum_{i \in \mathcal{I}} \bar{z}_{i} \leq(1-\|\bar{p}(0)\|-\|\bar{q}\|) I,
$$

which together with the above inequality (3.4) implies that $\|\bar{p}(0)\|+\|\bar{q}\|<1$. Hence there exists $\alpha>1$ such that $\|\alpha \bar{p}(0)\|+\|\alpha \bar{q}\|<1$ and $\alpha \bar{q} \in \operatorname{cl} Q(\bar{p}) \cap Z_{\mathcal{F}}$ (since the latter set is a cone). Consequently, from (3.2), (taking $(p, q) \in \Pi$ defined by $p(0)=\alpha \bar{p}(0), p(s)=\bar{p}(s)$ for $s \neq 0$ and $q=\alpha \bar{q}$ ) we deduce that:

$$
\alpha \bar{p}(0) \cdot \sum_{i \in \mathcal{I}}\left(\bar{x}_{i}(0)-e_{i}(0)\right)+\alpha \bar{q} \cdot \sum_{i \in \mathcal{I}} \bar{z}_{i} \leq \bar{p}(0) \cdot \sum_{i \in \mathcal{I}}\left(\bar{x}_{i}(0)-e_{i}(0)\right)+\bar{q} \cdot \sum_{i \in \mathcal{I}} \bar{z}_{i} .
$$

Dividing by $\bar{p}(0) \cdot \sum_{i \in I}\left(\bar{x}_{i}(0)-e_{i}(0)\right)+\bar{q} \cdot \sum_{i \in I} \bar{z}_{i}>0$ (by inequality (3.4)), we get $\alpha \leq 1$, which contradicts that $\alpha>1$.

Third, we show that $\sum_{i \in \mathcal{I}} \bar{z}_{i} \in-\boldsymbol{A}_{\mathcal{F}}$. From above, we have $\sum_{i \in \mathcal{I}} \bar{z}_{i} \in\left(\operatorname{cl} Q(\bar{p}) \cap Z_{\mathcal{F}}\right)^{o}=-\boldsymbol{A}_{\mathcal{F}}+\left(Z_{\mathcal{F}}\right)^{\perp}$ (by Lemma 4). Thus, there exists $a \in \boldsymbol{A}_{\mathcal{F}}$ such that $\sum_{i \in \mathcal{I}} \bar{z}_{i}+a \in\left(Z_{\mathcal{F}}\right)^{\perp}$ but $\sum_{i \in I} \bar{z}_{i}+a \in Z_{\mathcal{F}}$ (since $a \in \boldsymbol{A}_{\mathcal{F}} \subset Z_{\mathcal{F}}$ and $\bar{z}_{i} \in Z_{i} \subset Z_{\mathcal{F}}$ for each $\left.i \in \mathcal{I}\right)$. Hence $\sum_{i \in \mathcal{I}} \bar{z}_{i}+a \in\left(Z_{\mathcal{F}}\right)^{\perp} \cap Z_{\mathcal{F}}=\{0\}$. Therefore, $\sum_{i \in \mathcal{I}} \bar{z}_{i}=-a \in-\boldsymbol{A}_{\mathcal{F}}$.

Finally, we show that $\bar{q} \cdot \sum_{i \in I} \bar{z}_{i}=0$. We have $\bar{q} \cdot \sum_{i \in I} \bar{z}_{i} \leq 0$ since $\bar{q} \in \operatorname{cl} Q(\bar{p}) \cap Z_{\mathcal{F}}$ and $\sum_{i \in I} \bar{z}_{i} \in\left(\operatorname{cl} Q(\bar{p}) \cap Z_{\mathcal{F}}\right)^{o}$ (from above). Taking $(p, q)=(\bar{p}, 0) \in \Pi$ in (3.2) we deduce that $0 \leq \bar{q} \cdot \sum_{i \in I} \bar{z}_{i}$. Hence, $\bar{q} \cdot \sum_{i \in I} \bar{z}_{i}=0$.

Part (b). The equality $\sum_{i \in I} \overline{\bar{z}}_{i}=0$ is straightforward and, for all $i, V(\bar{p}) \overline{\bar{z}}_{i} \geq V(\bar{p}) \bar{z}_{i}$ (since $\overline{\bar{z}}_{i}-\bar{z}_{i}=\zeta_{i} \in$ $\{V(\bar{p}) \geq 0\})$. To show that, for all $i, \overline{\bar{z}}_{i} \in \widehat{Z}_{i} \subset Z_{i}^{r}$, it is sufficient to prove that

$$
(\overline{\bar{z}}, \bar{p}) \in K_{\underline{v}}=\left\{\left(z_{1}, \cdots, z_{I}, p\right) \in\left(\prod_{i} Z_{i}\right) \times B_{L}(0,1): \forall i, V(p) z_{i} \geq \underline{v}_{i},-\sum_{i \in \mathcal{I}} z_{i} \in \boldsymbol{A}_{\mathcal{F}}\right\} .
$$

Indeed, for all $i, \overline{\bar{z}}_{i}=\bar{z}_{i}+\zeta_{i} \in Z_{i}$ (since $\bar{z}_{i} \in Z_{i}^{r} \subset Z_{i}$ and $\zeta_{i} \in A Z_{i}$ ). Moreover, $V(\bar{p}) \overline{\bar{z}}_{i} \geq V(\bar{p}) \bar{z}_{i} \geq \underline{v}_{i}$ by Part (a). Finally, $\sum_{i \in I} \overline{\bar{z}}_{i}=0 \in \boldsymbol{A}_{\mathcal{F}}$. This ends the proof that $(\overline{\bar{z}}, \bar{p}) \in K_{\underline{v}}$.

We now show that $\bar{q} \cdot\left(\overline{\bar{z}}_{i}-\bar{z}_{i}\right)=\bar{q} \cdot \zeta_{i}=0$ for every $i \in \mathcal{I}$. Indeed, first $-\bar{q} \cdot \zeta_{i} \leq 0$ for every $i$ since $\bar{q} \in \operatorname{cl} Q(\bar{p}) \cap Z_{\mathcal{F}}=-\left(\boldsymbol{A}_{\mathcal{F}}+\left(Z_{\mathcal{F}}\right)^{\perp}\right)^{o}$ (by Lemma 4) and $\zeta_{i} \in A Z_{i} \cap\{V(\bar{p}) \geq 0\} \subset \boldsymbol{A}_{\mathcal{F}}$. Recalling that $\bar{q} \cdot \sum_{i \in I} \zeta_{i}=-\bar{q} \cdot \sum_{i \in I} \bar{z}_{i}=0$ from $\operatorname{Part}(a)$, we deduce that $\bar{q} \cdot \zeta_{i}=0$ for every $i \in I$.

Finally, for all $i,\left(\bar{x}_{i}, \overline{\bar{z}}_{i}\right) \in B_{i}^{r \varepsilon}(\bar{p}, \bar{q})$ since $\left(\bar{x}_{i}, \bar{z}_{i}\right) \in B_{i}^{r \varepsilon}(\bar{p}, \bar{q})$ (by 3.3)) and, from above $W(\bar{p}, \bar{q}) \bar{z}_{i} \leq$ $W(\bar{p}, \bar{q}) \overline{\bar{z}}_{i}$.

We now show that the Market Clearing Condition holds for the commodity markets.

Claim 3.3. $\sum_{i \in \mathcal{I}} \bar{x}_{i}=\sum_{i \in \mathcal{I}} e_{i}$.

Proof. We first prove that the equality holds at state $s=0$. If $\sum_{i \in \mathcal{I}} \bar{x}_{i}(0) \neq \sum_{i \in \mathcal{I}} e_{i}(0)$, we deduce from (3.2), (taking $(p, q) \in \Pi$ defined by $p(0)=\sum_{i \in \mathcal{I}}\left(\bar{x}_{i}(0)-e_{i}(0)\right) /\left\|\sum_{i \in \mathcal{I}}\left(\bar{x}_{i}(0)-e_{i}(0)\right)\right\|, p(s)=\bar{p}(s)$ for $s \neq 0$ and $q=0)$ that

$$
0<\left\|\sum_{i \in \mathcal{I}}\left(\bar{x}_{i}(0)-e_{i}(0)\right)\right\| \leq \bar{p}(0) \cdot \sum_{i \in \mathcal{I}}\left(\bar{x}_{i}(0)-e_{i}(0)\right)+\bar{q} \cdot \sum_{i \in \mathcal{I}} \bar{z}_{i}
$$

and in the exact same way as for inequality (3.4) in the proof of Claim 3.2 we obtain a contradiction. 
We now prove that the equality holds for all state $s \neq 0$. Suppose that, for some $s \neq 0, \sum_{i \in \mathcal{I}} \bar{x}_{i}(s) \neq \sum_{i \in \mathcal{I}} e_{i}(s)$. From (3.2), we deduce that $\|\bar{p}(s)\|=1$, hence $\varepsilon_{s}(\bar{p}, \bar{q})=1-\|\bar{p}(s)\|=0$, and

$$
0<\bar{p}(s) \cdot \sum_{i \in \mathcal{I}}\left(\bar{x}_{i}(s)-e_{i}(s)\right)
$$

Since $\left(\bar{x}_{i}, \overline{\bar{z}}_{i}\right) \in B_{i}^{r \varepsilon}(\bar{p}, \bar{q})$ (by Claim 3.2), and $\varepsilon_{s}(\bar{p}, \bar{q})=0$, we have $\bar{p}(s) \cdot\left(\bar{x}_{i}(s)-e_{i}(s)\right) \leq V_{s}(\bar{p}) \cdot \overline{\bar{z}}_{i}$ for all $i \in \mathcal{I}$, where $V_{s}(\bar{p})$ denotes the $s$-th row of the matrix $V(\bar{p})$. Summing up over $i$, and using the fact that $\sum_{i \in \mathcal{I}} \overline{\bar{z}}_{i}=0$ (by Claim 3.2) we get $\bar{p}(s) \cdot \sum_{i \in \mathcal{I}}\left(\bar{x}_{i}(s)-e_{i}(s)\right) \leq \sum_{i \in \mathcal{I}} V_{s}(\bar{p}) \cdot \overline{\bar{z}}_{i}=0$, a contradiction with the above strict inequality.

\subsection{The list $(\bar{x}, \overline{\bar{z}}, \bar{p}, \bar{q})$ is an equilibrium of $(\mathcal{E}, \mathcal{F})$}

We prepare the proof with the following claims.

Claim 3.4. $B_{i}^{r \varepsilon}(\bar{p}, \bar{q}) \cap\left(P_{i}^{r}(\bar{x}) \times Z_{i}^{r}\right)=\emptyset$.

Proof. $i$ From (3.3), one has $\breve{B}_{i}^{r \varepsilon}(\bar{p}, \bar{q}) \cap\left(P_{i}^{r}(\bar{x}) \times Z_{i}^{r}\right)=\emptyset$. Since $P_{i}^{r}$ has open values and since $B_{i}^{r \varepsilon}(\bar{p}, \bar{q})=$ $\operatorname{cl}_{i}^{r \varepsilon}(\bar{p}, \bar{q})$ (by Claim 3.1 since $(\bar{p}, \bar{q}) \in \Pi$ ), we deduce that $B_{i}^{r \varepsilon}(\bar{p}, \bar{q}) \cap\left(P_{i}^{r}(\bar{x}) \times Z_{i}^{r}\right)=\emptyset$.

Claim 3.5. $\varepsilon(\bar{p}, \bar{q})=0$, that is, $\|\bar{p}(0)\|+\|\bar{q}\|=1$, and $\|\bar{p}(s)\|=1$ for every $s \in \mathcal{S}$.

Proof. ¿From Claim 3.2, $\left(\bar{x}_{i}, \overline{\bar{z}}_{i}\right) \in B_{i}^{r \varepsilon}(\bar{p}, \bar{q})$ for each $i \in \mathcal{I}$, hence $\bar{p} \square\left(\bar{x}_{i}-e_{i}\right) \leq W(\bar{p}, \bar{q}) \overline{\bar{z}}_{i}+\varepsilon(\bar{p}, \bar{q})$. We now claim that the budget inequality is binding, that is

$$
\bar{p} \square\left(\bar{x}_{i}-e_{i}\right)=W(\bar{p}, \bar{q}) \overline{\bar{z}}_{i}+\varepsilon(\bar{p}, \bar{q}) \text { for all } i \in \mathcal{I} .
$$

Indeed, if it is not true then there exists $s \in \overline{\mathcal{S}}$ such that $\bar{p}(s) \cdot\left(\bar{x}_{i}(s)-e_{i}(s)\right)<W_{s}(\bar{p}, \bar{q}) \cdot \overline{\bar{z}}_{i}+\varepsilon_{s}(\bar{p}, \bar{q})$. From the Local Nonsatiation LNS, there exists $x_{i}^{n}(s) \rightarrow \bar{x}_{i}(s)$ such that $x_{i}^{n}:=\left(x_{i}^{n}(s), \bar{x}_{i}(-s)\right) \subset P_{i}^{r}(\bar{x})$ for all $n$. Then, it is possible to choose $n$ large enough so that $\left(x_{i}^{n}, \overline{\bar{z}}_{i}\right) \in B_{i}^{r \varepsilon}(\bar{p}, \bar{q})$, which together with $x_{i}^{n} \in P_{i}^{r}(\bar{x})$ contradicts the fact that $B_{i}^{r \varepsilon}(\bar{p}, \bar{q}) \cap\left(P_{i}^{r}(\bar{x}) \times Z_{i}^{r}\right)=\emptyset$ (by Claim 3.4).

Summing up over $i$ the equalities (3.5) and using the facts that $\sum_{i \in I} \overline{\bar{z}}_{i}=0$ (Claim 3.2) and $\sum_{i \in I} \bar{x}_{i}=\sum_{i \in I} e_{i}$ (Claim 3.3), we get $\operatorname{I\varepsilon }(\bar{p}, \bar{q})=0$. Hence $\varepsilon(\bar{p}, \bar{q})=0$.

We now show that $(\bar{x}, \overline{\bar{z}}, \bar{p}, \bar{q})$ is an equilibrium of $(\mathcal{E}, \mathcal{F})$. Since $\varepsilon(\bar{p}, \bar{q})=0$ (Claim 3.5) for every $i$, $B_{i}^{r \varepsilon}(\bar{p}, \bar{q})=B_{\mathcal{F}^{r}}^{i}(\bar{p}, \bar{q})$ and from Claims 3.2,3.3, and 3.4, we deduce that $(\bar{x}, \overline{\bar{z}}, \bar{p}, \bar{q})$ is an equilibrium of $\left(\mathcal{E}^{r}, \mathcal{F}^{r}\right)$. To show that it is an equilibrium of $(\mathcal{E}, \mathcal{F})$, we only have to prove that

$$
B_{\mathcal{F}}^{i}(\bar{p}, \bar{q}) \cap\left(P_{i}(\bar{x}) \times Z_{i}\right)=\emptyset \text { for every } i \in \mathcal{I} \text {. }
$$

Assume that it is not true, then for some $i$, there exists $\left(x_{i}, z_{i}\right) \in B_{\mathcal{F}}^{i}(\bar{p}, \bar{q}) \cap\left(P_{i}(\bar{x}) \times Z_{i}\right)$. ¿From the choice of of $r=\left(r_{1}, r_{2}\right)$ in the definition of the truncated economy, for all $i, \bar{x}_{i} \in \widehat{X}_{i} \subset \operatorname{int} B_{L}\left(0, r_{1}\right)$ (since $\bar{x}$ is an attainable allocation) and $\overline{\bar{z}}_{i} \in \widehat{Z}_{i} \subset \operatorname{int} B_{J}\left(0, r_{2}\right)$ (by Claim 3.2). Thus, for $t>0$ small enough, 
$\left(x_{i}^{t}, z_{i}^{t}\right):=\left(\bar{x}_{i}+t\left(x_{i}-\bar{x}_{i}\right), \overline{\bar{z}}_{i}+t\left(z_{i}-\overline{\bar{z}}_{i}\right)\right) \in X_{i}^{r} \times Z_{i}^{r}$, hence $\left(x_{i}^{t}, z_{i}^{t}\right) \in B_{\mathcal{F} r}^{i}(\bar{p}, \bar{q})$ (since $\left(x_{i}, z_{i}\right) \in B_{\mathcal{F}}^{i}(\bar{p}, \bar{q})$ and $\left.\left(\bar{x}_{i}, \bar{z}_{i}\right) \in B_{\mathcal{F}^{r}}^{i}(\bar{p}, \bar{q})\right)$. On the other hand, from the Local Nonsatiation Assumption LNS, for every $t \in(0,1]$, $\bar{x}_{i}+t\left(x_{i}-\bar{x}_{i}\right) \in P_{i}(\bar{x})$. Hence $\left(x_{i}^{t}, z_{i}^{t}\right) \in B_{\mathcal{F}^{r}}^{i}(\bar{p}, \bar{q}) \cap\left(P_{i}^{r}(\bar{x}) \times Z_{i}^{r}\right) \neq \emptyset$, in contradiction with the fact it is empty by Claim 3.4 .

\section{Appendix}

We modify the financial structure $\mathcal{F}$ by considering the reduced financial structure $\mathcal{F}_{\pi}$ which has the same payoff matrix as $\mathcal{F}$ and the portfolio sets $\pi Z_{i}(i \in \mathcal{I})$ where $\pi$ is the orthogonal projection mapping on the orthogonal space to $\boldsymbol{L}_{\mathcal{F}}:=\boldsymbol{A}_{\mathcal{F}} \cap-\boldsymbol{A}_{\mathcal{F}}$. We recall that $Z_{\mathcal{F}}:=<\cup_{i} Z_{i}>, Z_{\mathcal{F}_{\pi}}:=<\cup_{i} \pi Z_{i}>$ and the definition of $\mathcal{F}_{\pi}$ can be summarized by

$$
\begin{gathered}
\mathcal{F}_{\pi}=\left(V(p),\left(\pi Z_{i}\right)_{i}\right), \\
\text { where } \pi=\operatorname{proj}_{\left(\boldsymbol{L}_{\mathcal{F}}\right)^{\perp}}, \boldsymbol{L}_{\mathcal{F}}:=\boldsymbol{A}_{\mathcal{F}} \cap-\boldsymbol{A}_{\mathcal{F}} \text {, and } \boldsymbol{A}_{\mathcal{F}}:=\sum_{i \in \mathcal{I}}\left(\boldsymbol{A} Z_{i} \cap\{V(p) \geq 0\}\right) \subset Z_{\mathcal{F}},
\end{gathered}
$$

In the following, we will use extensively the following properties ${ }^{8}$ of the linear mapping $\pi$ : for all $(p, q, z) \in$ $\mathbb{R}^{L} \times \mathbb{R}^{J} \times \mathbb{R}^{J}$,

$$
q \cdot \pi z=\pi q \cdot z, V(p) \pi z=V(p) z, \text { hence } W(p, q) \pi z=W(p, \pi q) z
$$

\subsection{Proof of Lemma 1}

We prepare the proof with two claims.

Claim 4.1. $Q_{\mathcal{F}_{\pi}}(p) \cap Z\left(\mathcal{F}_{\pi}\right) \subset Q_{\mathcal{F}}(p) \cap Z_{\mathcal{F}}$ for every $p \in \mathbb{R}^{L}$.

Proof. We first claim that $Q_{\mathcal{F}_{\pi}}(p) \cap Z\left(\mathcal{F}_{\pi}\right) \subset Q_{\mathcal{F}_{\pi}}(p) \cap \operatorname{Im} \pi \subset Q_{\mathcal{F}}(p)$ for every $p \in \mathbb{R}^{L}$.

The first inclusion is a consequence of the fact that $Z\left(\mathcal{F}_{\pi}\right) \subset \operatorname{Im} \pi$. We prove the second inclusion by contradiction. Assume that there is some $q \in Q_{\mathcal{F}_{\pi}}(p) \cap \operatorname{Im} \pi$ such that $q \notin Q_{\mathcal{F}}(p)$. Then there exists $i \in \mathcal{I}$ and $\zeta_{i} \in A Z_{i}$ such that $W(p, q) \zeta_{i}>0$. But $\pi \zeta_{i} \in \pi\left(A Z_{i}\right) \subset \boldsymbol{A}\left(\pi Z_{i}\right)$ (from Rockafellar (1970)) and by (4.1) (since $q \in \operatorname{Im} \pi$ implies $q=\pi q), W(p, q)\left(\pi \zeta_{i}\right)=W(p, q) \zeta_{i}>0$, which contradicts the fact that $q \in Q_{\mathcal{F}_{\pi}}(p)$. This ends the proof of the two inclusions.

We end the proof of Claim 4.1 by showing that $Z\left(\mathcal{F}_{\pi}\right) \subset Z_{\mathcal{F}}$. Indeed, let $y \in Z\left(\mathcal{F}_{\pi}\right)$, then $y=\pi z$ for some $z \in Z_{\mathcal{F}}$ and $y=\pi z=\pi z-z+z \in \operatorname{ker} \pi+Z_{\mathcal{F}} \subset Z_{\mathcal{F}}$ since $\operatorname{ker} \pi=\boldsymbol{L}_{\mathcal{F}} \subset Z_{\mathcal{F}}$.

Claim 4.2. $\sum_{i \in \mathcal{I}}\left(\pi Z_{i} \cap\left\{V(p) \geq v_{i}\right\}\right) \subset \sum_{i \in \mathcal{I}}\left(Z_{i} \cap\left\{V(p) \geq v_{i}\right\}\right)$ for all $v=\left(v_{i}\right)_{i \in I} \in\left(\mathbb{R}^{S}\right)^{I}$ and all $p \in \mathbb{R}^{L}$.

\footnotetext{
${ }^{8}$ The first equality comes from the fact that $\pi q \cdot \pi z=\pi q \cdot z$, since $\pi q \in \operatorname{Im} \pi$ and $z-\pi z \in \operatorname{ker} \pi=(\operatorname{Im} \pi)^{\perp}$ since $\pi$ is an orthogonal projection mapping; then by symmetry $q \cdot \pi z=\pi q \cdot \pi z=\pi q \cdot z$. The second one holds since $z-\pi z \in \operatorname{ker} \pi=\boldsymbol{L}_{\mathcal{F}}$ and $\boldsymbol{L}_{\mathcal{F}}:=\boldsymbol{A}_{\mathcal{F}} \cap-\boldsymbol{A}_{\mathcal{F}} \subset\{V(p) \geq 0\} \cap-\{V(p) \geq 0\}=\operatorname{ker} V(p)$.
} 
Proof. The result is a consequence of the two following inclusions

$$
\sum_{i \in \mathcal{I}}\left(\pi Z_{i} \cap\left\{V(p) \geq v_{i}\right\}\right) \subset \operatorname{ker} \pi+\sum_{i \in \mathcal{I}}\left(Z_{i} \cap\left\{V(p) \geq v_{i}\right\}\right) \subset \sum_{i \in \mathcal{I}}\left(Z_{i} \cap\left\{V(p) \geq v_{i}\right\}\right) .
$$

To prove the first inclusion, let $y=\sum_{i \in I} y_{i}$ such that $y_{i}=\pi z_{i}$ for some $z_{i} \in Z_{i}$ and $V(p) y_{i} \geq v_{i}$. Then $y=\sum_{i \in \mathcal{I}} y_{i}=\sum_{i \in \mathcal{I}}\left(\pi z_{i}-z_{i}\right)+\sum_{i \in \mathcal{I}} z_{i} \in \operatorname{ker} \pi+\sum_{i \in \mathcal{I}}\left(Z_{i} \cap\left\{V(p) \geq v_{i}\right\}\right)$ since $\pi z_{i}-z_{i} \in \operatorname{ker} \pi$ and $V(p) z_{i}=V(p) \pi z_{i}=V(p) y_{i} \geq v_{i}$, from the properties (4.1) of the mapping $\pi$.

To prove the second inclusion, from the definition of the asymptotic cone in the convex case it suffices to show that $\operatorname{ker} \pi \subset A\left(\sum_{i \in \mathcal{I}}\left(Z_{i} \cap\left\{V(p) \geq v_{i}\right\}\right)\right)$ and that the convex set $\sum_{i \in \mathcal{I}}\left(Z_{i} \cap\left\{V(p) \geq v_{i}\right\}\right)$ is closed. From Rockafellar (1970), if the sets $C_{k}(k \in K)$ are polyhedral convex sets in $\mathbb{R}^{n}$, then $\sum_{k \in K} C_{k}$ is also a polyhedral convex set (hence is closed) and $\sum_{k \in K} \boldsymbol{A} C_{k}=\boldsymbol{A}\left(\sum_{k \in K} C_{k}\right)$. Since $Z_{i}(i \in I)$ is a polyhedral convex set (by Assumption FN1), the set $\left.Z_{i} \cap\left\{V(p) \geq v_{i}\right)\right\}$ is also a polyhedral convex set, hence $\sum_{i \in \mathcal{I}}\left(Z_{i} \cap\left\{V(p) \geq v_{i}\right\}\right)$ is a polyhedral set and it is closed. Recalling that $\operatorname{ker} \pi=\boldsymbol{L}_{\mathcal{F}} \subset \boldsymbol{A}_{\mathcal{F}}$, from the previously mentioned result we get

$$
\begin{aligned}
\operatorname{ker} \pi=\boldsymbol{L}_{\mathcal{F}} \subset \boldsymbol{A}_{\mathcal{F}}=\sum_{i \in \mathcal{I}} \boldsymbol{A} Z_{i} \cap\{V(p) \geq 0\} & =\sum_{i \in \mathcal{I}} \boldsymbol{A}\left(Z_{i} \cap\left\{V(p) \geq v_{i}\right\}\right) \\
& =\boldsymbol{A}\left(\sum_{i \in \mathcal{I}}\left(Z_{i} \cap\left\{V(p) \geq v_{i}\right\}\right)\right) .
\end{aligned}
$$

We are now ready to give the proof of Lemma 1.

0. $\mathcal{F}_{\pi}$ satisfies F0. The financial structure $\mathcal{F}$ satisfies F0, that is, the set $\boldsymbol{A}_{\mathcal{F}}:=\sum_{i \in \mathcal{I}}\left(A Z_{i} \cap\{V(p) \geq 0\}\right)$ does not depend on $p$. Clearly, $\mathcal{F}_{\pi}$ will also satisfy $\mathbf{F 0}$ if we show that $\boldsymbol{A}_{\mathcal{F}_{\pi}}:=\sum_{i \in \mathcal{I}}\left(\boldsymbol{A} \pi Z_{i} \cap\{V(p) \geq 0\}\right)=\pi\left(\boldsymbol{A}_{\mathcal{F}}\right)$. We recall that, for all $i, \boldsymbol{A} \pi Z_{i}=\pi \boldsymbol{A} Z_{i}$ since $Z_{i}$ is a polyhedral convex set (see Rockafellar (1970)). To prove the first inclusion, let $y \in \boldsymbol{A}_{\mathcal{F}_{\pi}}$, then $y=\sum_{i \in \mathcal{I}} y_{i}$ for some $y_{i}$ such that $y_{i}=\pi z_{i}$ for some $z_{i} \in \boldsymbol{A} Z_{i}$ and $V(p) y_{i} \geq 0$. But $V(p) z_{i}=V(p) \pi z_{i}=V(p) y_{i} \geq 0$ by (4.1), hence $y \in \pi\left(\boldsymbol{A}_{\mathcal{F}}\right)$. Conversely, let $y \in \pi\left(\boldsymbol{A}_{\mathcal{F}}\right)$, then $y=\pi\left(\sum_{i \in I} z_{i}\right)$ for some $\left.z_{i} \in \boldsymbol{A} Z_{i} \cap\{V(p) \geq 0\}\right)$. But $\pi z_{i} \in \pi \boldsymbol{A} Z_{i}=\boldsymbol{A} \pi Z_{i}$ and $V(p) \pi z_{i}=V(p) z_{i} \geq 0$ from the properties (4.1) of the mapping $\pi$. Thus $y=\sum_{i \in I} \pi z_{i} \in \boldsymbol{A}_{\mathcal{F}_{\pi}}$.

1. $\mathcal{F}_{\pi}$ satisfies $\mathbf{F 1}$ and FN1. Indeed, $\pi Z_{i}$ is a polyhedral convex set, since $Z_{i}$ is a polyhedral convex set and $\pi$ is linear (see for example Rockafellar (1970)) and $\pi Z_{i}$ contains 0 since $Z_{i}$ contains 0.

2. $\mathcal{F}_{\pi}$ satisfies F2. Let $q \in \operatorname{cl} Q_{\mathcal{F}_{\pi}}(p) \cap Z\left(\mathcal{F}_{\pi}\right) \backslash\{0\}$ and recall that $\operatorname{cl} Q_{\mathcal{F}_{\pi}}(p) \cap Z\left(\mathcal{F}_{\pi}\right) \backslash\{0\} \subset \operatorname{cl} Q_{\mathcal{F}}(p) \cap Z_{\mathcal{F}} \backslash\{0\}$ by Claim 4.1. Since $\mathcal{F}$ satisfies $\mathbf{F 2}$, for all $i \in \mathcal{I}$, there exists $z_{i} \in Z_{i}$ such that $q \cdot z_{i}<0$. But $\pi z_{i} \in \pi Z_{i}$ and $q \cdot \pi z_{i}=q \cdot z_{i}<0$ (from the properties (4.1) of the mapping $\pi$ since $q \in Z\left(\mathcal{F}_{\pi}\right) \subset \operatorname{Im} \pi$ ), thus $\mathcal{F}_{\pi}$ satisfies $\mathbf{F} 2$.

3. $\mathcal{F}_{\pi}$ satisfies F3. Taking $v_{i}=0$ for every $i$ in Claim 4.2, and in (4.2), we deduce that

$$
\boldsymbol{A}_{\mathcal{F}_{\pi}}=\boldsymbol{A}\left(\sum_{i \in \mathcal{I}}\left(\pi Z_{i} \cap\{V(p) \geq 0\}\right)\right) \subset \boldsymbol{A}\left(\sum_{i \in \mathcal{I}}\left(Z_{i} \cap\{V(p) \geq 0\}\right)\right)=\boldsymbol{A}_{\mathcal{F}}
$$

Consequently, $\boldsymbol{L}_{\mathcal{F}_{\pi}} \subset \boldsymbol{L}_{\mathcal{F}}$. Moreover, $\boldsymbol{L}_{\mathcal{F}_{\pi}} \subset \boldsymbol{A}_{\mathcal{F}_{\pi}} \subset \sum_{i \in \mathcal{I}}\left(\pi Z_{i} \cap\{V(p) \geq 0\}\right) \subset \operatorname{Im} \pi$ and $\boldsymbol{L}_{\mathcal{F}}=\operatorname{ker} \pi$ (from the definition of the mapping $\pi$ ). Consequently $\boldsymbol{L}_{\mathcal{F}_{\pi}} \subset \boldsymbol{L}_{\mathcal{F}} \cap \operatorname{Im} \pi=\operatorname{ker} \pi \cap \operatorname{Im} \pi=\{0\}$. This ends the proof of Lemma 1. 


\subsection{Proof of Lemma 2}

Let $(\bar{p}, \bar{q}, \bar{x}, \bar{z})$ be an equilibrium of the economy $\left(\mathcal{E}, \mathcal{F}_{\pi}\right)$. We first claim that there exists $z_{i}^{*} \in Z_{i}(i \in \mathcal{I})$ such that $V(\bar{p}) z_{i}^{*}=V(\bar{p}) \bar{z}_{i}$ for all $i$ and $\sum z_{i}^{*}=0$. Indeed, by Claim 4.2, taking $v_{i}:=V(\bar{p}) \bar{z}_{i}$ for all $i$, one gets

$$
0=\sum_{i \in \mathcal{I}} \bar{z}_{i} \in \sum_{i \in \mathcal{I}}\left(\pi Z_{i} \cap\left\{V(\bar{p}) \geq V(p) \bar{z}_{i}\right\}\right) \subset \sum_{i \in \mathcal{I}}\left(Z_{i} \cap\left\{V(\bar{p}) \geq V(\bar{p}) \bar{z}_{i}\right\}\right) .
$$

Hence $0=\sum_{i \in I} z_{i}^{*}$ for some $z_{i}^{*} \in Z_{i}$ such that $V(\bar{p}) z_{i}^{*} \geq V(\bar{p}) \bar{z}_{i}$ for every $i$. But $\sum_{i \in I} V(\bar{p})\left(z_{i}^{*}-\bar{z}_{i}\right)=0$ since $\sum_{i \in I}\left(z_{i}^{*}-\bar{z}_{i}\right)=0$ and we conclude that $V(\bar{p})\left(z_{i}^{*}-\bar{z}_{i}\right)=0$ for every $i$. This ends the proof of the claim.

We will show that $\left(\bar{p}, \pi \bar{q}, \bar{x}, z^{*}\right)$ is an equilibrium of $(\mathcal{E}, \mathcal{F})$. We first prove that $\left(\bar{x}_{i}, z_{i}^{*}\right) \in B_{\mathcal{F}}^{i}(\bar{p}, \pi \bar{q})$ for all $i$. Since $\left(\bar{x}_{i}, \bar{z}_{i}\right) \in B_{\mathcal{F}_{\pi}}^{i}(\bar{p}, \bar{q})$ it suffices to show that, for all $i, z_{i}^{*} \in Z_{i}$ and $W(\bar{p}, \pi \bar{q}) z_{i}^{*}=W(\bar{p}, \bar{q}) \bar{z}_{i}$. In view of the previous claim, $V(\bar{p}) z_{i}^{*}=V(\bar{p}) \bar{z}_{i}$ for all $i$ and it only remains to show that $\pi \bar{q} \cdot z_{i}^{*}=\bar{q} \cdot \bar{z}_{i}$ for all $i$. But, with the Market Clearing Condition $\sum z_{i}^{*}=0$ proved in the above claim, it suffices to show that $\pi \bar{q} \cdot z_{i}^{*} \geq \bar{q} \cdot \bar{z}_{i}$ for all $i$. Indeed, suppose that for some $i$ one has $-\pi \bar{q} \cdot z_{i}^{*}>-\bar{q} \cdot \bar{z}_{i}$. But this implies that $W(\bar{p}, \bar{q}) \pi z_{i}^{*}>W(\bar{p}, \bar{q}) \bar{z}_{i}$, with $\pi z_{i}^{*} \in \pi Z_{i}$; indeed, from above and the properties (4.1) of the mapping $\pi$, one has $-\bar{q} \cdot \pi z_{i}^{*}=-\pi \bar{q} \cdot z_{i}^{*}>-\bar{q} \cdot \bar{z}_{i}$ and $V(\bar{p}) \pi z_{i}^{*}=V(\bar{p}) z_{i}^{*}=V(\bar{p}) \bar{z}_{i}$. In other words, the $i$-th agent has an arbitrage opportunity in the financial economy $\left(\mathcal{E}, \mathcal{F}_{\pi}\right)$, which is impossible when $(\bar{p}, \bar{q}, \bar{x}, \bar{z})$ is an equilibrium of $\left(\mathcal{E}, \mathcal{F}_{\pi}\right)$ under Assumption $\mathbf{C}$ (see, for example, Angeloni and Cornet (2006)).

We end the proof by showing that $B_{\mathcal{F}}^{i}(\bar{p}, \pi \bar{q}) \cap\left[P_{i}(\bar{x}) \times Z_{i}\right]=\emptyset$ for all $i \in \mathcal{I}$. Suppose that for some $i$ there exists $\left(x_{i}, z_{i}\right) \in B_{\mathcal{F}}^{i}(\bar{p}, \pi \bar{q}) \cap\left[P_{i}(\bar{x}) \times Z_{i}\right]$. We then deduce that $\left(x_{i}, \pi z_{i}\right) \in B_{\mathcal{F}_{\pi}}^{i}(\bar{p}, \bar{q})$ since $\left(x_{i}, z_{i}\right) \in B_{\mathcal{F}}^{i}(\bar{p}, \pi \bar{q})$ and $W(\bar{p}, \pi \bar{q}) z_{i}=W(\bar{p}, \bar{q}) \pi z_{i}$, from the properties (4.1) of the mapping $\pi$. But $\left(x_{i}, \pi z_{i}\right) \in B_{\mathcal{F}_{\pi}}^{i}(\bar{p}, \bar{q})$, together with $\left(x_{i}, \pi z_{i}\right) \in P_{i}(\bar{x}) \times \pi Z_{i}$ contradicts the fact that $(\bar{p}, \bar{q}, \bar{x}, \bar{z})$ is an equilibrium of $\left(\mathcal{E}, \mathcal{F}_{\pi}\right)$.

\subsection{Proof of Lemma 3}

We prepare the proof of Lemma 3 with two claims. We let $S_{\ell}(0,1):=\left\{y \in \mathbb{R}^{\ell}:\|y\|=1\right\}$ and for every $s \in S$

$P_{s}:=\left\{p(s) \in \mathbb{R}^{\ell}: \exists i \in \mathcal{I}, \exists x \in \mathcal{A}(\mathcal{E}), \forall x_{i}^{\prime}(s),\left[\left(x_{i}^{\prime}(s), x_{i}(-s)\right) \in P_{i}(x) \Rightarrow p(s) \cdot x_{i}^{\prime}(s) \geq p(s) \cdot x_{i}(s) \geq p(s) \cdot e_{i}(s)\right]\right\}$

Claim 4.3. The set $P:=\Pi_{s \in \mathcal{S}} P_{s}$ is closed.

Proof. For every $s \in S$, let $\left(p^{n}(s)\right)_{n}$ be a sequence in $P_{s}$ such that $p^{n}(s) \rightarrow p(s)$. For every $n \in \mathbb{N}$, the property defining $P_{s}$ is satisfied for some agent $i_{s}^{n} \in \mathcal{I}$. Since there is a finite number of agents, by eventually considering a subsequence, we can assume there exists some agent $i_{s} \in \mathcal{I}$ (independent of $n$ ), say $i_{s}=1$, such that, for all $n$, there exists $x^{n} \in \mathcal{A}(\mathcal{E})$ and $\left(x_{1}^{\prime}(s), x^{n}(-s)\right) \in P_{1}\left(x^{n}\right)$ implies $p^{n}(s) \cdot x_{1}^{\prime}(s) \geq$ $p^{n}(s) \cdot x_{1}^{n}(s) \geq p^{n}(s) \cdot e_{1}(s)$. Since $\mathcal{A}(\mathcal{E})$ is compact (by Assumption $\mathbf{C}$ ), without any loss of generality, we can assume that $x^{n} \rightarrow x \in \mathcal{A}(\mathcal{E})$.

We show that $p(s) \in P_{s}$. Indeed, let $i=1$, let $x=\lim _{n \rightarrow \infty} x_{n}$ as defined above, and let $\left(x_{1}^{\prime}(s), x_{1}(-s)\right) \in$ $P_{1}(x)$. By Assumption FNo, $P_{1}$ has an open graph, hence for $n$ large enough, $\left(x_{1}^{\prime}(s), x_{1}^{n}(-s)\right) \in P_{1}\left(x^{n}\right)$ since $\left(x_{1}^{\prime}(s), x_{1}^{n}(-s)\right) \rightarrow\left(x_{1}^{\prime}(s), x_{1}(-s)\right)$ and $x^{n} \rightarrow x$. From above, we deduce that $p^{n}(s) \cdot x_{1}^{\prime}(s) \geq p^{n}(s) \cdot x_{1}^{n}(s) \geq$ 
$p^{n}(s) \cdot e_{1}(s)$. Passing to the limit, when $n \rightarrow \infty$, we get $p(s) \cdot x_{1}^{\prime}(s) \geq p(s) \cdot x_{1}(s) \geq p(s) \cdot e_{1}(s)$, hence $p(s) \in P_{s}$.

Claim 4.4. There exists $\varepsilon>0$ such that $p(s) \cdot v \geq \varepsilon\|p(s)\| \quad$ for all $p \in P$ and all $s \in \mathcal{S}$.

Proof. We start by proving that, for every $p(s) \in P_{s} \cap S_{\ell}(0,1), p(s) \cdot v>0$. Indeed, if $p(s) \in P_{s}$, there exist $i_{s} \in \mathcal{I}$, say $i_{s}=1$, and $x \in \mathcal{A}(\mathcal{E})$ such that $\left(x_{1}^{\prime}(s), x_{1}(-s)\right) \in P_{1}(x)$ implies $p(s) \cdot x_{1}^{\prime}(s) \geq p(s) \cdot x_{1}(s) \geq$ $p(s) \cdot e_{1}(s)$. Since $e_{1} \in \operatorname{int} X_{1}$ (by the Survival Assumption in $\mathbf{C}$ ) and $\|p(s)\|=1$ (since $p(s) \in S_{\ell}(0,1)$ ), there exists $y_{1} \in X_{1}$ such that $p(s) \cdot x_{1}(s) \geq p(s) \cdot e_{1}(s)>p(s) \cdot y_{1}(s)$. But, for every $t>0,\left(x_{1}(s)+t v, x_{1}(-s)\right) \in P_{1}(x)$ (from the Desirability Assumption in FN). Moreover, since $P_{1}(x)$ is open (by Assumption C), it is possible to choose $x_{1}^{\prime}(s)$ such that $\left(x_{1}^{\prime}(s)+t v, x_{1}(-s)\right) \in P_{1}(x)$ and $p(s) \cdot x_{1}(s)>p(s) \cdot x_{1}^{\prime}(s){ }^{9}$. Since $p(s) \in P_{s}$ we deduce that

$$
p(s) \cdot\left[x_{1}^{\prime}(s)+t v\right] \geq p(s) \cdot x_{1}(s), \text { that is } t p(s) \cdot v \geq p(s) \cdot x_{1}(s)-p(s) \cdot x_{1}^{\prime}(s)>0 .
$$

Hence we have proved that, for every $p(s) \in P_{s} \cap S_{\ell}(0,1)$, there exists $\varepsilon_{p}>0$ such that $p(s) \cdot v>\varepsilon_{p}$. This implies that

$$
P_{s} \cap S_{\ell}(0,1) \subset \bigcup\left\{p(s) \in S_{\ell}(0,1): p(s) \cdot v>\varepsilon_{p}\right\} .
$$

Since $P_{s}$ is closed (by Claim 4.3) the set $P_{s} \cap S_{\ell}(0,1)$ is compact and there exist finitely many prices $p_{1}, \ldots, p_{r}$ such that

$$
P_{s} \cap S_{\ell}(0,1) \subset \bigcup_{k=1}^{r}\left\{p(s) \in S_{\ell}(0,1): p(s) \cdot v>\varepsilon_{p_{k}}\right\} .
$$

We let $\varepsilon(s):=\min \left\{\varepsilon_{p_{1}}, \ldots, \varepsilon_{p_{r}}\right\}$ and $\varepsilon:=\min \{\varepsilon(s): s \in \mathcal{S}\}$. Then, for every $p(s) \in P_{s} \backslash\{0\}, p(s) /\|p(s)\| \in P_{s}$ (noticing that $P_{s}$ is a cone) hence $(p(s) /\|p(s)\|) \cdot v>\varepsilon(s)>0$ and, for all $p \in P$ and all $s \in \mathcal{S}, p(s) \cdot v \geq$ $\varepsilon\|p(s)\|$.

Proof of Lemma 3. We choose $\varepsilon>0$ as in Claim 4.4 and we show that every equilibrium $(\bar{p}, \bar{q}, \bar{x}, \bar{z})$ of $\left(\mathcal{E}, \mathcal{F}^{\varepsilon}\right)$ such that $\|\bar{p}(s)\|=1$ for $s \in \mathcal{S}$, is also an equilibrium of $(\mathcal{E}, \mathcal{F})$. First we claim that $\bar{p} \in P$, that is $\bar{p}(s) \in P_{s}$ for every $s \in \mathcal{S}$. From the Financial Market Clearing Condition $\sum_{i \in \mathcal{I}} \bar{z}_{i}=0$ we deduce that $V_{s}^{\varepsilon}(\bar{p}) \cdot\left(\sum_{i \in I} \bar{z}_{i}\right)=0$, hence there exists $i_{s} \in \mathcal{I}$, say $i=1$, such that $V_{s}^{\varepsilon}(\bar{p}) \cdot \bar{z}_{1} \geq 0$. We prove that $\bar{p}(s) \in P_{s}$ by showing that it satisfies the condition defining $P_{s}$ with $i=1$ and $\bar{x}=\left(\bar{x}_{i}\right)_{i} \in \mathcal{A}(\mathcal{E})$. Indeed, let $x_{1}^{\prime}:=\left(x_{1}^{\prime}(s), \bar{x}_{1}(-s)\right) \in P_{1}(\bar{x})$. First we have $\bar{p}(s) \cdot x_{1}^{\prime}(s) \geq \bar{p}(s) \cdot \bar{x}_{1}(s)$. Otherwise

$$
\bar{p}(s) \cdot\left(x_{1}^{\prime}(s)-e_{1}(s)\right)<\bar{p}(s) \cdot\left(\bar{x}_{1}(s)-e_{1}(s)\right) \leq V_{s}^{\varepsilon}(\bar{p}) \cdot \bar{z}_{1} .
$$

Consequently, $\left(x_{1}^{\prime}, \bar{z}_{1}\right) \in B_{\mathcal{F}^{\varepsilon}}^{1}(\bar{p}, \bar{q}) \cap\left[P_{1}(\bar{x}) \times Z_{1}\right]$, which contradicts the fact that $(\bar{p}, \bar{q}, \bar{x}, \bar{z})$ is an equilibrium of $\left(\mathcal{E}, \mathcal{F}^{\varepsilon}\right)$. Second we have $\bar{p}(s) \cdot \bar{x}_{1}(s) \geq \bar{p}(s) \cdot e_{1}(s)$. Otherwise $\bar{p}(s) \cdot\left(\bar{x}_{1}(s)-e_{1}\right)<0$. Hence for $t>0$ small enough

$$
\bar{p}(s) \cdot\left(\left(\bar{x}_{1}(s)+t v\right)-e_{1}\right)<0 \leq V_{s}^{\varepsilon}(\bar{p}) \cdot \bar{z}_{1}
$$

\footnotetext{
${ }^{9}$ Indeed, since $P_{1}(x)$ is open, there exists $\lambda \in(0,1]$ such that $\lambda\left(x_{1}(s)+t v, x_{1}(-s)\right)+(1-\lambda)\left(y_{1}(s)+t v, x_{1}(-s)\right)=\left(\lambda x_{1}(s)+(1-\right.$ ג) $\left.\left.y_{1}(s)+t v, x_{1}(-s)\right)\right) \in P_{1}(x)$, then choose $x_{1}^{\prime}(s)=\lambda x_{1}(s)+(1-\lambda) y_{1}(s)$, then one has $p(s) \cdot x_{1}^{\prime}(s)<p(s) \cdot x_{1}(s)$.
} 
Consequently, from the desirability assumption, we get $\left(\left(\bar{x}_{1}(s)+t v, \bar{x}_{1}(-s)\right), \bar{z}_{1}\right) \in B_{\mathcal{F}^{\varepsilon}}^{1}(\bar{p}, \bar{q}) \cap\left[P_{1}(\bar{x}) \times Z_{1}\right]$, which contradicts the fact that $(\bar{p}, \bar{q}, \bar{x}, \bar{z})$ is an equilibrium in $\left(\mathcal{E}, \mathcal{F}^{\varepsilon}\right)$. This ends the proof that $\bar{p} \in P$.

Since $\bar{p} \in P$, and $\|\bar{p}(s)\|=1$ for $s \in \mathcal{S}$, we deduce that $\bar{p}(s) \cdot v \geq \varepsilon\|\bar{p}(s)\|=\varepsilon$, by Claim 4.4. Consequently, $V^{\varepsilon}(\bar{p})=V(\bar{p})$, for every $i \in \mathcal{I}, B_{\mathcal{F} \varepsilon}^{i}(\bar{p}, \bar{q})=B_{\mathcal{F}}^{i}(\bar{p}, \bar{q})$ and clearly $(\bar{p}, \bar{q}, \bar{x}, \bar{z})$ is an equilibrium for $(\mathcal{E}, \mathcal{F})$.

\subsection{Proof of Lemma 4}

Part (a). Let $p \in \mathbb{R}^{L}$. The set $Q(p)$ is obviously a cone, and we now show that it is convex by contradiction. Suppose that there exist $q_{1}, q_{2}$ in $Q(p), \alpha \in(0,1)$ such that $\alpha q_{1}+(1-\alpha) q_{2} \notin Q(p)$. Then there exists $i \in \mathcal{I}$ and there exists $\zeta \in \boldsymbol{A} Z_{i}$ such that $W\left(p, \alpha q_{1}+(1-\alpha) q_{2}\right) \zeta>0$. Hence

$$
\text { either }\left\{\begin{array} { l } 
{ - ( \alpha q _ { 1 } + ( 1 - \alpha ) q _ { 2 } ) \cdot \zeta > 0 } \\
{ V ( p ) \zeta \geq 0 }
\end{array} \text { or } \left\{\begin{array}{l}
-\left(\alpha q_{1}+(1-\alpha) q_{2}\right) \cdot \zeta \geq 0 \\
V(p) \zeta>0
\end{array}\right.\right.
$$

In the first case, we conclude that either $-q_{1} \cdot \zeta>0$ or $-q_{2} \cdot \zeta>0$ which, together with $V(p) \zeta \geq 0$, implies that $W\left(p, q_{1}\right) \zeta>0$ or $W\left(p, q_{1}\right) \zeta>0$, contradicting the fact that $q_{1}$ and $q_{2}$ are both in $Q(p)$. Similarly, in the second case, we conclude that either $-q_{1} \cdot \zeta \geq 0$ or $-q_{2} \cdot \zeta \geq 0$ which, together with $V(p) \zeta>0$, contradicts the fact that $q_{1}$ and $q_{2}$ are both in $Q(p)$.

Part (b). Step 1. For every $p$, the set $\boldsymbol{A}_{\mathcal{F}}(p):=\sum_{i \in \mathcal{I}}\left(\boldsymbol{A} Z_{i} \cap\{V(p) \geq 0\}\right)$ is closed. The set $\boldsymbol{A}_{\mathcal{F}}(p)$ is simply denoted $\boldsymbol{A}$ hereafter. From Debreu (1959), it suffices to show that the convex cones $\boldsymbol{A} Z_{i} \cap\{V(p) \geq 0\}$ are positively semi-independent. ${ }^{10}$ Indeed, let $\zeta_{i} \in A Z_{i} \cap\{V(p) \geq 0\}$ for each $i$ such that $\sum_{i \in \mathcal{I}} \zeta_{i}=0$. Then $\zeta_{1}=-\sum_{i \neq 1} \zeta_{i} \in \boldsymbol{A} \cap-\boldsymbol{A}=\{0\}$ by Assumption F3. Hence $\zeta_{1}=0$ and similarly, $\zeta_{i}=0$ for every $i$.

Step 2. $-\operatorname{int}\left(\boldsymbol{A}^{o}\right) \subset Q(p)$. Suppose there exists $q \in-\operatorname{int}\left(\boldsymbol{A}^{o}\right)$ and $q \notin Q(p)$. Then there exist $i \in \mathcal{I}$ and $\zeta \in \boldsymbol{A} Z_{i}$ such that $W(p, q) \zeta>0$. Thus $\zeta \in \boldsymbol{A} Z_{i} \cap\{V(p) \geq 0\} \backslash\{0\} \subset \boldsymbol{A} \backslash\{0\}$. Recalling that $\boldsymbol{A}$ is a pointed closed convex cone (by assumption $\mathbf{F 3}$ and Step 1), from Rockafellar (1970), we have $\emptyset \neq \operatorname{int}\left(\boldsymbol{A}^{o}\right)=\{q \in$ $\left.\mathbb{R}^{J}, q \cdot c<0, \forall c \in \boldsymbol{A} \backslash\{0\}\right\}$. Consequently, $-q \cdot \zeta<0$ since $-q \in \operatorname{int}\left(\boldsymbol{A}^{o}\right)$ and $\zeta \in \boldsymbol{A} \backslash\{0\}$, a contradiction with $-q \cdot \zeta \geq 0($ since $W(p, q) \zeta>0)$.

Step 3. $-Q(p)^{o}=\boldsymbol{A}$. First we prove that $-Q(p)^{o} \subset \boldsymbol{A}$. Since $\operatorname{int}\left(\boldsymbol{A}^{o}\right) \neq \emptyset$, we have $\boldsymbol{A}^{o}=\operatorname{cl} \operatorname{int}\left(\boldsymbol{A}^{o}\right)$ (see Rockafellar (1970)). From Step 2, one gets $-Q(p)^{o} \subset\left[\operatorname{int}\left(\boldsymbol{A}^{o}\right)\right]^{o}=\left[\operatorname{cl} \operatorname{int}\left(\boldsymbol{A}^{o}\right)\right]^{o}=\left(\boldsymbol{A}^{o}\right)^{o}=\boldsymbol{A}$ from the Bipolar Theorem (see Rockafellar (1970)) since $\boldsymbol{A}$ is a closed convex cone (by Step 1). Second, we show that $\boldsymbol{A} \subset-Q(p)^{o}$. Let $\zeta \in \boldsymbol{A}$, then $\zeta=\sum_{i \in I} \zeta_{i}$ for some $\zeta_{i} \in A Z_{i} \cap\{V(p) \geq 0\}$. Consequently, $-q \cdot \zeta_{i} \leq 0$ for every $q \in Q(p)$ by definition of arbitrage-free prices. Hence $\zeta=\sum_{i \in I} \zeta_{i} \in-Q(p)^{o}$.

Step 4. The set $-\boldsymbol{A}+\left(Z_{\mathcal{F}}\right)^{\perp}$ is closed. Indeed, it suffices to show that the closed convex cones $-\boldsymbol{A}$ (which is closed by Step1) and $\left(Z_{\mathcal{F}}\right)^{\perp}$ (a vector space, hence closed) are positively independent. This is clearly the case since $-\boldsymbol{A} \subset Z_{\mathcal{F}}$.

\footnotetext{
${ }^{10} \mathrm{~A}$ finite collection $\left\{C_{i}, i \in \mathcal{I}\right\}$ of nonempty convex cones in $\mathbb{R}^{n}$ is positively semi-independent if $c_{i} \in C_{i}$, for all $i \in \mathcal{I}$ and $\sum_{i \in \mathcal{I}} c_{i}=0$, imply that for all $i \in \mathcal{I}, c_{i}=0$.
} 
Step 5. We are ready to conclude. First we note that for two closed convex cones $M$ and $N$ of $\mathbb{R}^{J}$, one has $(M \cap N)^{o}=\operatorname{cl}\left(M^{o}+N^{o}\right)$, hence from the above steps one gets

$$
\left(\operatorname{cl} Q(p) \cap Z_{\mathcal{F}}\right)^{o}=\operatorname{cl}\left((\operatorname{cl} Q(p))^{o}+Z_{\mathcal{F}}^{o}\right)=\operatorname{cl}\left(Q(p)^{o}+\left(Z_{\mathcal{F}}\right)^{\perp}\right)=\operatorname{cl}\left(-\boldsymbol{A}+\left(Z_{\mathcal{F}}\right)^{\perp}\right)=-\boldsymbol{A}+\left(Z_{\mathcal{F}}\right)^{\perp} .
$$

Part (c). Assume $K_{v}$ is not bounded. Then there exist sequences $\left(p^{n}\right)_{n} \subset B_{L}(0,1),\left(z_{i}^{n}\right)_{n} \subset Z_{i}(i \in \mathcal{I})$ such that for all $n$ and all $i, V\left(p^{n}\right) z_{i}^{n} \geq v_{i},-\sum_{i \in I} z_{i}^{n} \in \boldsymbol{A}_{\mathcal{F}}$, and $\sum_{i \in \mathcal{I}}\left\|z_{i}^{n}\right\| \underset{n \rightarrow \infty}{\longrightarrow}+\infty$. Passing to a subsequence if necessary, we can assume that $\left(p^{n}\right)_{n}$ converges to some $p \in B_{L}(0,1)$ and that, for each $i$, the bounded sequence $\left(z_{i}^{n} / \sum_{k \in I}\left\|z_{k}^{n}\right\|\right)_{n}$ converges to some $\zeta_{i}$. The vector $\zeta_{i}$ belongs to $A Z_{i}$ since $z_{i}^{n} \in Z_{i}$ for every $n$ and $1 / \sum_{k \in I}\left\|z_{k}^{n}\right\| \underset{n \rightarrow \infty}{\longrightarrow} 0$. Moreover, for every $n$, we have $V\left(p^{n}\right)\left(z_{i}^{n} / \sum_{k \in I}\left\|z_{k}^{n}\right\|\right) \geq v_{i} / \sum_{k \in I}\left\|z_{k}^{n}\right\|$. Passing to the limit, we obtain $V(p) \zeta_{i} \geq 0$ (since $V$ is continuous). Recalling that, for every $n,-\sum_{i \in I} z_{i}^{n} \in \boldsymbol{A}_{\mathcal{F}}$, we get $-\sum_{i \in \mathcal{I}}\left(z_{i}^{n} / \sum_{k \in \mathcal{I}}\left\|z_{k}^{n}\right\|\right) \underset{n \rightarrow \infty}{\longrightarrow}-\sum_{i \in \mathcal{I}} \zeta_{i} \in \boldsymbol{A}_{\mathcal{F}}$ since $\boldsymbol{A}_{\mathcal{F}}$ is closed by Part (b). Recalling that for each $i$, $\zeta_{i} \in \boldsymbol{A Z}_{i} \cap\{V(p) \geq 0\} \subset \boldsymbol{A}_{\mathcal{F}}$ we conclude that $\sum_{i \in I} \zeta_{i} \in \boldsymbol{A}_{\mathcal{F}} \cap-\boldsymbol{A}_{\mathcal{F}}=\{0\}$ (by Assumption F3). Hence $\zeta_{i} \in \boldsymbol{A}_{\mathcal{F}}$ for each $i$ and $\sum_{i \in \mathcal{I}} \zeta_{i}=0$, which implies that $\zeta_{1}=-\sum_{i \neq 1} \zeta_{i} \in \boldsymbol{A}_{\mathcal{F}} \cap-\boldsymbol{A}_{\mathcal{F}}=\{0\}$, and similarly $\zeta_{i}=0$ for every $i$. But $z_{i}^{n} / \sum_{k \in \mathcal{I}}\left\|z_{k}^{n}\right\| \underset{n \rightarrow \infty}{\longrightarrow} \zeta_{i}$, hence $1=\sum_{i \in \mathcal{I}} \frac{\left\|z_{i}^{n}\right\|}{\sum_{k \in I}\left\|z_{k}^{n}\right\|} \underset{n \rightarrow \infty}{\longrightarrow} \sum_{i \in \mathcal{I}}\left\|\zeta_{i}\right\|=0$, a contradiction.

\subsection{Proof of Lemma 5}

First, we claim that, for all $i \in \mathcal{I}$, the correspondence $B_{i}^{r \varepsilon}$ from $\Pi$ to $X_{i}^{r} \times Z_{i}^{r}$ is lower semicontinuous, has a closed graph, and has closed convex values. Indeed, from Claim 3.1, $B_{i}^{r \varepsilon}$ is the closure of $\breve{B}_{i}^{r \varepsilon}$ on $\Pi$. We then notice that $\breve{B}_{i}^{r \varepsilon}$ has an open graph, hence is lower semicontinuous. Consequently, $B_{i}^{r \varepsilon}$, which is the closure of a lower semicontinuous correspondence, is lower semicontinuous. The fact that $B_{i}^{r \varepsilon}$ has a closed graph and closed convex values is immediate.

The correspondence $\Phi_{0}$ has clearly an open graph thus it is lower semicontinuous and one easily checks that it has convex values. Let $i \neq 0,(\bar{p}, \bar{q}, \bar{x}, \bar{z}) \in \Pi \times \prod_{k \in I} X_{k}^{r} \times Z_{k}^{r}$, and let $V$ be an open set in $X_{i}^{r} \times Z_{i}^{r}$ such that $\Phi_{i}(\bar{p}, \bar{q}, \bar{x}, \bar{z}) \cap V \neq \emptyset$. We distinguish two cases:

1. $\left(\bar{x}_{i}, \bar{z}_{i}\right) \notin B_{i}^{r \varepsilon}(\bar{p}, \bar{q})$. Then $\left(\bar{p}, \bar{q}, \bar{x}_{i}, \bar{z}_{i}\right) \notin G\left(B_{i}^{r \varepsilon}\right)$ and since $B_{i}^{r \varepsilon}$ has a closed graph there exists an open neighborhood $W$ of $\left(\bar{p}, \bar{q}, \bar{x}_{i}, \bar{z}_{i}\right)$ in $\Pi \times\left(X_{i}^{r} \times Z_{i}^{r}\right)$ such that $W \cap G\left(B_{i}^{r \varepsilon}\right)=\emptyset$. On the other hand, $V$ is open in $X_{i}^{r} \times Z_{i}^{r}$ and satisfies $B_{i}^{r \varepsilon}(\bar{p}, \bar{q}) \cap V \neq \emptyset$ (because $\Phi_{i}(\bar{p}, \bar{q}, \bar{x}, \bar{z}) \cap V \neq \emptyset$ and $\Phi_{i}(\bar{p}, \bar{q}, \bar{x}, \bar{z}) \subset B_{i}^{r \varepsilon}(\bar{p}, \bar{q})$ ). Since $B_{i}^{r \varepsilon}$ is lower semicontinuous we conclude that there exists an open neighborhood $O$ of $(\bar{p}, \bar{q})$ in $\Pi$ such that for every $(p, q) \in O, B_{i}^{r \varepsilon}(p, q) \cap V \neq \emptyset$. Then $U:=\left[W \times\left(\prod_{k \neq i} X_{k}^{r} \times Z_{k}^{r}\right)\right] \cap\left[O \times\left(\prod_{k} X_{k}^{r} \times Z_{k}^{r}\right)\right]$ is an open neighborhood of $(\bar{p}, \bar{q}, \bar{x}, \bar{z})$ and for every $(p, q, x, z) \in U$ one has $\left(p, q, x_{i}, z_{i}\right) \in W \cap\left[O \times\left(X_{i}^{r} \times Z_{i}^{r}\right)\right]$, hence $\Phi_{i}(p, q, x, z) \cap V=B_{i}^{r \varepsilon}(p, q) \cap V \neq \emptyset$.

2. $\left(\bar{x}_{i}, \bar{z}_{i}\right) \in B_{i}^{r \varepsilon}(\bar{p}, \bar{q})$. Note that $\breve{B}_{i}^{r \varepsilon} \cap\left(P_{i}^{r} \times Z_{i}^{r}\right)$ is lower semicontinuous since $\breve{B}_{i}^{r \varepsilon}$ has an open graph and $P_{i}^{r} \times Z_{i}^{r}$ is lower semicontinuous. Since $\breve{B}_{i}^{r \varepsilon}(\bar{p}, \bar{q}) \cap\left(P_{i}^{r}(\bar{x}) \times Z_{i}^{r}\right) \cap V=\Phi_{i}(\bar{p}, \bar{q}, \bar{x}, \bar{z}) \cap V \neq \emptyset$, we conclude that there exists an open neighborhood $T$ of $(\bar{p}, \bar{q}, \bar{x}, \bar{z})$ in $\Pi \times \prod_{k \in I} X_{k}^{r} \times Z_{k}^{r}$ such that for every $(p, q, x, z) \in T$ one has $\breve{B}_{i}^{r \varepsilon}(p, q) \cap\left(P_{i}^{r}(x) \times Z_{i}^{r}\right) \cap V \neq \emptyset$. Consequently, $B_{i}^{r \varepsilon}(p, q) \cap V \neq \emptyset$ (because $\left.\breve{B}_{i}^{r \varepsilon}(p, q) \subset B_{i}^{r \varepsilon}(p, q)\right)$ and $\Phi_{i}(p, q, x, z) \cap V \neq \emptyset$ for every $(p, q, x, z) \in T$. This ends the proof of the lower semicontinuity of $\Phi_{i}$. 
The convexity of the values of $\Phi_{i}$ is a consequence of the convexity of $\breve{B}_{i}^{r \varepsilon}(p, q), B_{i}^{r \varepsilon}(p, q), Z_{i}^{r}$ and the convexity of the values of $P_{i}^{r}$, by assumptions $\mathbf{C}$ and $\mathbf{F 1}$.

\section{References}

Angeloni, L., Cornet, B., 2006. Existence of financial equilibria in a multi-period stochastic economy. Mathematical Economics 8 , $1-31$.

Aouani, Z., Cornet, B., 2008a. Characterizing reduced financial structures, Working Paper.

Aouani, Z., Cornet, B., 2008b. Reduced equivalent form of a financial structure, Working Paper.

Balasko, Y., Cass, D., Siconolfi, P., 1990. The structure of financial equilibrium with exogenous yields: The case of restricted participation. Journal of Mathematical Economics 19, 195-216.

Bergstrom, T. C., 1976. How to discard "free disposability" - at no cost. Journal of Mathematical Economics 3, $131-134$.

Bich, P., Cornet, B., 2004. Fixed-point-like theorems on subspaces. Fixed Point Theory and Applications 3, $159-171$.

Bich, P., Cornet, B., 2009. Existence of pseudo-equilibria in a financial economy. Journal of Fixed Point Theory and Applications, forthcoming.

Cass, D., 1984. Competitive equilibrium with incomplete financial markets, CARESS Working Paper.

Cass, D., 2006. Competitive equilibrium with incomplete financial markets. Journal of Mathematical Economics 42, 384-405.

Cornet, B., Gopalan, R., 2010. Arbitrage and equilibrium with portfolio constraints. Economic Theory, forthcoming.

Debreu, G., 1959. Theory of Value. Yale University Press.

Duffie, D., 1987. Stochastic equilibria with incomplete financial markets. Journal of Economic Theory 41, 404-416.

Duffie, D., Shafer, W., 1985. Equilibrium in incomplete markets I: A basic model of generic existence. Journal of Mathematical Economics 14, 285-300.

Duffie, D., Shafer, W., 1986. Equilibrium in incomplete markets II: Generic existence in stochastic economies. Journal of Mathematical Economics 15, 199-216.

Elsinger, H., Summer, M., 2001. Arbitrage and optimal portfolio choice with financial constraints, Working Paper, Austrian Central Bank.

Gale, D., Mas-Colell, A., 1975. An equilibrium existence Theorem for a general model without ordered preferences. Journal of Mathematical Economics 2, 9-15.

Gale, D., Mas-Colell, A., 1979. Corrections to an equilibrium existence Theorem for a general model without ordered preferences. Journal of Mathematical Economics 6, 297-298.

Geanakoplos, J. D., Polemarchakis, H., 1986. Uncertainty, information and communication: Essays in honor of Kenneth J. Arrow. Vol. III. Heller et al. Ed. Cambridge University Press, Cambridge, Ch. Existence, regularity and constrainted suboptimality of competitive allocations when the asset market is incomplete, pp. 65-95.

Geanakopolos, J. D., Shafer, W., 1990. Solving systems of simultaneous equations in economics. Journal of Mathematical Economics 19, 69-93.

Hahn, G., Won, D. C., 2007. Constrained asset markets, available at SSRN: http://ssrn.com/abstract=1021631.

Hart, O., 1975. On the optimality of equilibrium when the market structure is incomplete. Journal of Economic Theory 11, 418443.

Hirsch, M., Magill, M., Mas-Colell, A., 1990. A geometric approach to a class of equilibrium existence theorems. Journal of Mathematical Economics 19, 95-106.

Husseini, S. Y., Lasry, J., Magill, M., 1990. Existence of equilibrium with incomplete markets. Journal of Mathematical Economics 19, 39-67.

Martins-da-Rocha, F., Triki, L., 2005. Equilibria in exchange economies with financial constraints: Beyond the Cass-trick, Working Paper, University of Paris 1.

Polemarchakis, H. M., Siconolfi, P., 1997. Generic existence of competitive equilibria with restricted participation. Journal of Mathematical Economics 28, 289-311. 
Radner, R., 1972. Existence of equilibrium of plans, prices, and price expectations. Econometrica 40, $289-303$.

Rockafellar, R. T., 1970. Convex Analysis. Princeton University Press.

Siconolfi, P., 1989. Equilibrium with asymmetric constraints on portfolio holdings and incomplete financial markets. In: M. Gale-

otti, L. Geronazzo and F. Gori, eds, Non-Linear Dynamics in Economics and Social Sciences, Societa' Pitagora. pp. $271-292$.

Werner, J., 1985. Equilibrium in economies with incomplete financial markets. Journal of Economic Theory 36, 110-119. 